\title{
KUPERBERG AND TURAEV-VIRO INVARIANTS IN UNIMODULAR CATEGORIES
}

\author{
FRANCESCO COSTANTINO, NATHAN GEER, BERTRAND PATUREAU-MIRAND, \\ AND VLADIMIR TURAEV
}

\begin{abstract}
We give a categorical setting in which Penrose graphical calculus naturally extends to graphs drawn on the boundary of a handlebody. We use it to introduce invariants of 3-manifolds presented by Heegaard splittings. We recover Kuperberg invariants when the category arises from an involutory Hopf algebra and Turaev-Viro invariants when the category is semi-simple and spherical.
\end{abstract}

\section{CONTENTS}

1. Introduction

2. Main results and open problems

3. The algebraic setup

4. Proof of Theorem 2.1

5. Invariants of bichrome graphs and 3-manifolds 14

6. Hopf Algebras and the Kuperberg invariants 19

7. Proof of Theorem $2.7 \quad 26$

References

\section{INTRODUCTION}

A remarkable achievement of the low-dimensional topology in the last 30 years was a discovery of deep relations between topology and the theory of monoidal (tensor) categories. This development was initiated by V. Jones' introduction of his famous knot polynomial; by now it englobes many aspects of low-dimensional topology including 3-manifold invariants, representations of mapping class groups of surfaces, topological quantum field theories in dimensions 2 and 3, etc. In particular, it was shown that monoidal categories satisfying certain conditions and carrying appropriate additional structures give rise to topological invariants of 3 -dimensional manifolds, see [26, 27]. This has instigated extensive research in the theory of monoidal categories aiming at construction (and eventually classification) of monoidal categories with required properties. At the same time, this development has inspired

This work is supported by the NSF FRG Collaborative Research Grant DMS-1664387. 
a number of parallel approaches not involving monoidal categories but using related algebraic objects. One such approach is due to G. Kuperberg [16] who derived invariants of 3-manifolds from involutory Hopf algebras. The initial aim of this paper was to recover Kuperberg's invariants in terms of monoidal categories. To this end we introduce a new construction of 3-manifold invariants from monoidal categories. We show that our method produces both the Kuperberg invariants and the standard Turaev-Viro invariants. Other generalizations of Kuperberg invariants were considered by Kashaev and Virelizier in [15].

The first main result of our paper is a construction - in a general categorical setting - of an invariant of graphs on the boundary of a handlebody. With

some additional categorical structure we extend this invariant to bichrome graphs which split as the union of two subgraphs, the blue and the red. Here the red part has the following key property: any edge of the graph can be slid over a red curve. Our definition of a 3-manifold invariant uses Heegaard decompositions of 3-manifolds as unions of two handlebodies glued along their common boundary. Such a decomposition is encoded via a redcolored set of disjoint simple closed curves on the boundary of one handlebody forming a complete set of meridians for the second handlebody.

On the algebraic side, we use two main tools - the modified traces (mtraces) and the chromatic morphisms. The m-traces generalize the usual trace of endomorphisms of objects of a monoidal category to situations where the standard trace vanishes. The m-traces first appeared in [10, 11, 14] and have been successfully used to produce 3-manifold invariants, see for example [4, 7, 13]. The chromatic morphisms are introduced here.

\section{MAin RESUlts AND OPEN PROBLEMS}

2.1. The invariant $F^{\prime}$. We start with notation used in the paper, for details see Section 3 , Let $\mathscr{C}$ be a pivotal $\mathbb{k}$-category, where $\mathbb{k}$ is a field. Let $F$ be the Penrose functor (defined using the Penrose graphical calculus) from the category of planar $\mathscr{C}$-colored ribbon graphs to $\mathscr{C}$, see for example [14]. Let t be a modified trace (for shorteness, an m-trace) on an ideal $\mathcal{I}$ in $\mathscr{C}$. The trace $\mathrm{t}$ induces an invariant $F^{\prime}$ of $\mathcal{I}$-colored spherical graphs, see [14]. This invariant can be computed by composing $\mathrm{t}$ and $F$ on cutting presentations of the graphs (see Formula (8)).

We assume the m-trace t to be non-degenerate in the sense that for any object $P \in \mathcal{I}$, the pairing

$$
\operatorname{Hom}_{\mathscr{C}}(\mathbb{1}, P) \times \operatorname{Hom}_{\mathscr{C}}(P, \mathbb{1}) \rightarrow \mathbb{k}, \quad(x, y) \mapsto \mathrm{t}_{P}(x y)
$$


is non-degenerate. Pick a basis $\left\{x_{i}\right\}$ of $\operatorname{Hom}_{\mathscr{C}}(\mathbb{1}, P)$ and let $\left\{y_{i}\right\}$ be the dual basis of $\operatorname{Hom}_{\mathscr{C}}(P, \mathbb{1})$ with respect to the above pairing. Set

$$
\Omega_{P}=\sum_{i} x_{i} \otimes_{\mathbb{k}} y_{i} \in \operatorname{Hom}_{\mathscr{C}}(\mathbb{1}, P) \otimes_{\mathbb{k}} \operatorname{Hom}_{\mathscr{C}}(P, \mathbb{1})
$$

where $\otimes_{\mathbb{k}}$ is the usual tensor product of vector spaces over $\mathbb{k}$. It is a standard fact from linear algebra that $\Omega_{P}$ does not dependent on the choice of the basis $\left\{x_{i}\right\}$ of $\operatorname{Hom}_{\mathscr{C}}(\mathbb{1}, P)$.

By a multi-handlebody we mean a disjoint union of a finite number of oriented 3-dimensional handlebodies. A $\mathscr{C}$-colored ribbon graph on a multihandlebody $H$ is a finite graph embedded in $\partial H$ whose every edge is colored with an object of $\mathscr{C}$ and every vertex is thickened in $\partial H$ to a coupon colored with a morphism of $\mathscr{C}$. All coupons have a top and a bottom sides which in the pictures will be the horizontal sides. Since our graphs are drawn on a surface they have a natural framing and therefore can be considered as ribbon graphs in the usual sense. When all the colors of a $\mathscr{C}$-colored ribbon graph are in the ideal $\mathcal{I}$ we say that the graph is $\mathcal{I}$-colored. Let $\mathscr{H}_{\mathcal{I}}$ be the class of all pairs $(H, \Gamma)$ where $H$ is a multi-handlebody and $\Gamma$ is a non-empty $\mathcal{I}$-colored graph on $H$. In the sequel we extend the colorings of coupons multilinearly. In particular, this allows us to color an ordered matching pair of coupons with the morphism $\Omega_{P}=\sum_{i} x_{i} \otimes_{\mathbb{k}} y_{i}$ as above. We represent such a pair of coupons with their adjacent edges by the following figure:
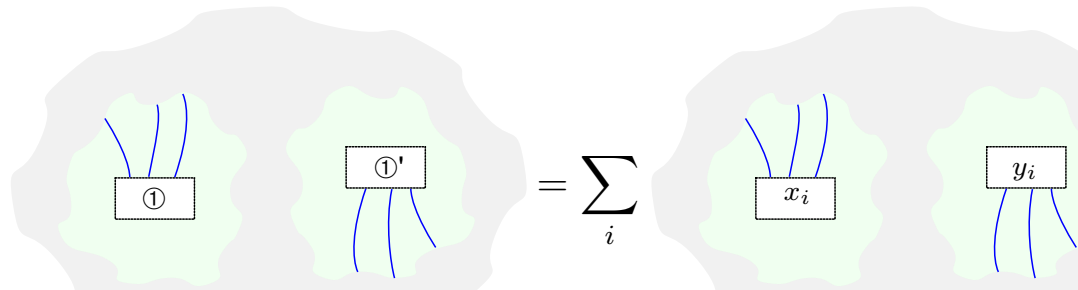

We now define a cutting operation on colored graphs. Let $(H, \Gamma) \in \mathscr{H}_{\mathcal{I}}$ and let $D \subset H$ be an oriented properly embedded disc whose boundary $\partial D \subset \partial H$ does not meet the coupons of $\Gamma$ and intersects the edges of $\Gamma$ transversely in a non-empty set. Cutting $(H, \Gamma)$ along $D$ we obtain a new multi-handlebody graph $\left(\operatorname{cut}_{D}(H), \operatorname{cut}_{D}(\Gamma)\right) \in \mathscr{H}_{\mathcal{I}}$ where $\operatorname{cut}_{D}(\Gamma)$ is obtained by cutting the edges of $\Gamma$ intersecting $\partial D$ and then joining the cut points into two new coupons in $\partial\left(\operatorname{cut}_{D}(H)\right)$ (one on each side of the cut). The coupons 
are colored as in Formula (2), see the following figure:

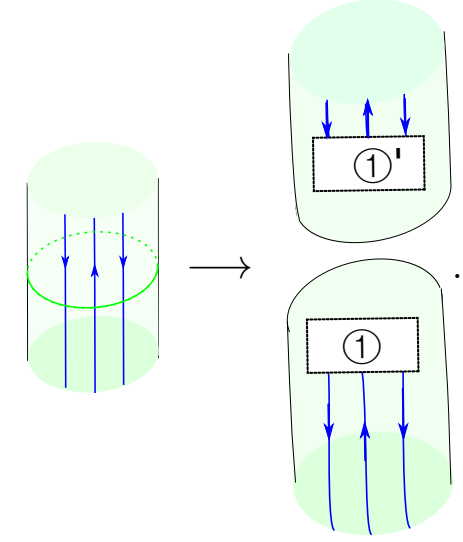

Note that $H$ and $\operatorname{cut}_{D}(H)$ can have different numbers of connected components and the orientation of $\operatorname{cut}_{D}(H)$ is induced by the one of $H$. The cutting of $(H, \Gamma)$ at $D$ is not determined by these data uniquely as it depends on the order in the set $\Gamma \cap \partial D$ compatible with the cyclic order in the oriented circle $\partial D$.

Below we define an invariant $F^{\prime}$ of $\mathcal{I}$-colored spherical graphs, see Formula (8). The following theorem (proved in Section 4) extends $F^{\prime}$ to $\mathscr{H}_{\mathcal{I}}$.

Theorem 2.1. Let $\mathscr{C}$ be a pivotal $\mathbb{k}$-category equipped with an ideal $\mathcal{I}$ in $\mathscr{C}$ and a non-degenerate $m$-trace on $\mathcal{I}$. Then there exists a unique mapping $F^{\prime}: \mathscr{H}_{\mathcal{I}} \rightarrow \mathbb{k}$ satisfying the following four conditions.

(1) Invariance: The element $F^{\prime}(H, \Gamma)$ of $\mathbb{k}$ depends only on the orientation preserving diffeomorphism class of $(H, \Gamma) \in \mathscr{H}_{\mathcal{I}}$.

(2) Spherical case: For any $\mathcal{I}$-colored ribbon graph $\left(B^{3}, \Gamma\right)$ on the 3 ball $B^{3}$, we have $F^{\prime}\left(B^{3}, \Gamma\right)=F^{\prime}(\Gamma)$.

(3) Disjoint union: For any $\left(H_{1}, \Gamma_{1}\right),\left(H_{2}, \Gamma_{2}\right) \in \mathscr{H}_{\mathcal{I}}$ we have

$$
F^{\prime}\left(H_{1} \sqcup H_{2}, \Gamma_{1} \sqcup \Gamma_{2}\right)=F^{\prime}\left(H_{1}, \Gamma_{1}\right) F^{\prime}\left(H_{2}, \Gamma_{2}\right) .
$$

(4) Cutting: Cutting any $(H, \Gamma) \in \mathscr{H}_{\mathcal{I}}$ along a 2-disc $D$ as above, we always have $F^{\prime}\left(\operatorname{cut}_{D}(H)\right.$, $\left._{D}(\Gamma)\right)=F^{\prime}(H, \Gamma)$.

The assumptions of Theorem 2.1 are rather mild. By Theorem 5.5 of [12] (see also [11]) the categories arising in the following settings satisfy these assumptions: representations of factorizable ribbon Hopf algebras, finite groups and their quantum doubles, Lie (super)algebras, the $(1, p)$ minimal model in conformal field theory, and quantum groups at a root of unity.

Following the ideas of [8], we futher extend $F^{\prime}$ to so-called bichrome handlebody graphs. A bichrome handlebody graph is a graph on the boundary of a multi-handlebody which is split as a disjoint union of two subgraphs, the blue and the red. The blue subgraph is supposed to be $\mathscr{C}$-colored while 
the red subgraph is supposed to be a disjoint union of simple closed unoriented curves (which are not required to be $\mathscr{C}$-colored). We refer to these curves as red circles. We call a bichrome handlebody graph admissible if its blue subgraph is $\mathcal{I}$-colored and meets each connected component of the multi-handlebody at a non-empty set.

Definition 2.2. Let $G$ be an object of $\mathcal{I}$. Set $\Lambda=\sum_{i} x_{i} y_{i} \in \operatorname{End}_{\mathscr{C}}\left(G \otimes G^{*}\right)$ where $\Omega_{G \otimes G^{*}}=\sum x_{i} \otimes_{\mathbb{k}} y_{i}$. A chromatic morphism for $G$ is a morphism $\widetilde{d}: G \otimes G \rightarrow G \otimes G$ such that

$$
\left(\operatorname{Id}_{G} \otimes \overleftarrow{\mathrm{ev}}_{G} \otimes \operatorname{Id}_{G}\right) \circ(\Lambda \otimes \widetilde{d}) \circ\left(\operatorname{Id}_{G} \otimes \overrightarrow{\operatorname{coev}}_{G} \otimes \operatorname{Id}_{G}\right)=\operatorname{Id}_{G \otimes G}
$$

This equality is represented pictorially as

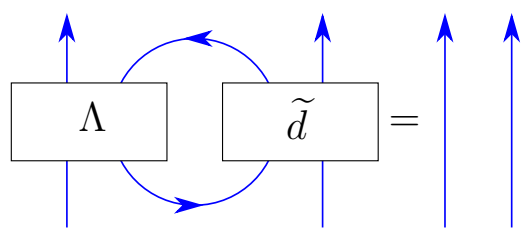

where all blue strands are colored by $G$. We utilize the word chromatic here because the morphism $\widetilde{d}$ is used to change a red circle into a blue graph, see Theorem 2.3 below. In the setting of Hopf algebras this transformation corresponds to evaluation of the integral on the red circle (see Section 6).

A generator of an ideal $\mathcal{I}$ is an object $G \in \mathcal{I}$ such that for any $P \in \overline{\mathcal{I}}$,

$$
\operatorname{Id}_{P}=\sum_{j \in J} f_{j} g_{j}
$$

for some morphisms $f_{j}: G \rightarrow P, g_{j}: P \rightarrow G$ numerated by a finite set $J$. As we will see below, in the setting of Hopf algebras such a generator is a projective generator.

Let $\widetilde{d}$ be a chromatic morphism for a generator $G$ of an ideal $\mathcal{I}$. If $P \in \mathcal{I}$ and $\operatorname{Id}_{P}=\sum_{j} f_{j} g_{j}$ as in Formula (4), then we set

$$
\widetilde{d}_{P}=\sum_{j \in J}\left(\operatorname{Id}_{G} \otimes f_{j}\right) \widetilde{d}\left(\operatorname{Id}_{G} \otimes g_{j}\right): G \otimes P \rightarrow G \otimes P .
$$

The following theorem is proved in Section 5.3 .

Theorem 2.3. Let $\mathscr{C}$ be a pivotal $\mathbb{k}$-category equipped with a non-degenerate $m$-trace on an ideal $\mathcal{I}$ and a chromatic morphism $\widetilde{d}$ on a generator $G \in \mathcal{I}$. Then there exists a unique extension of $F^{\prime}: \mathscr{H}_{\mathcal{I}} \rightarrow \mathbb{k}$ to admissible bichrome handlebody graphs which is preserved under the following transformation making a red circle blue in the presence of a nearby blue edge colored with an 
object $P \in \mathcal{I}$ as shown in the figure:

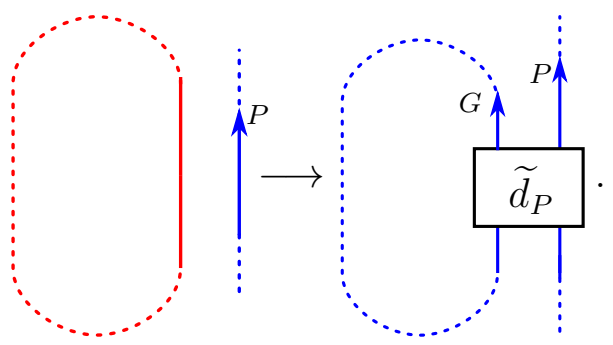

Moreover, if $(H, \Gamma)$ is a bichrome handlebody graph then $F^{\prime}(H, \Gamma)$ only depends on the orientation preserving diffeomorphism class of $(H, \Gamma)$.

Later we will see that $F^{\prime}$ is also invariant under sliding an edge of $\Gamma$ over a red circle, see Proposition 5.4 .

2.2. The invariant $\mathcal{K}$. Let $\mathscr{C}$ be a pivotal $\mathbb{k}$-category equipped with a nondegenerate $\mathrm{m}$-trace $\mathrm{t}$ on an ideal $\mathcal{I}$ and a chromatic morphism on a generator $G$ of $\mathcal{I}$. Since $\mathrm{t}$ is non-degenerate, there exists a morphism $h: G \rightarrow G$ such that $\mathrm{t}_{G}(h) \neq 0$. By renormalizing the $\mathrm{m}$-trace we can assume $\mathrm{t}_{G}(h)=1$. Let $\mathbf{O}_{G}$ be the ribbon graph in $\mathbb{R}^{2}$ formed by the braid closure of the coupon filled with $h$. Consider the bichrome handlebody graph $\left(B^{3}, \mathbf{O}_{G}\right)$ where $\mathbf{O}_{G}$ is colored in blue and viewed as a graph on $S^{2}=\partial B^{3}$. Clearly, $F^{\prime}\left(B^{3}, \mathbf{O}_{G}\right)=F^{\prime}\left(\mathbf{O}_{G}\right)=\mathrm{t}_{G}(h)=1$.

Let $M$ be a closed connected oriented 3-manifold. A Heegaard diagram of $M$ is a prescription for a Heegaard splitting $M=H_{\alpha} \cup_{\Sigma} H_{\beta}$ determined by upper and lower reducing sets of bounding circles $\left\{\alpha_{i}\right\}$ and $\left\{\beta_{i}\right\}$ in $\Sigma=\partial H_{\alpha}=\partial H_{\beta}$, see Section 5 for details. A Heegaard diagram as above determines an admissible bichrome handlebody graph on $H_{\alpha}$ where the red subgraph is the set $\cup_{i} \beta_{i} \subset \partial H_{\alpha}$ and the blue subgraph is $\mathbf{O}_{G}$ embedded in a small disc in $\partial H_{\alpha}$. We call this bichrome handlebody graph a bichrome diagram for $M$, see Figure 1 for an example.

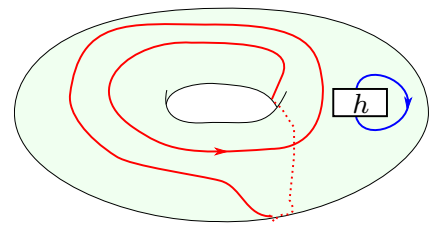

Figure 1. Bichrome diagram for the lens space $L(2,1)$.

We state the main theorem of the paper proved in Section 5.3.

Theorem 2.4. If $(H, \Gamma)$ is a bichrome diagram for a closed connected oriented 3-manifold $M$, then $F^{\prime}(H, \Gamma) \in \mathbb{k}$ depends only of the diffeomorphism class of $M$. 
We denote the invariant of Theorem 2.4 by $\mathcal{K}_{\mathscr{C}}(M)$. Let us now discuss some examples.

2.3. Hopf algebras and Kuperberg's Invariant. We use standard terminology from the theory of Hopf algebras, for details see Section 6. Let $A$ be a finite dimensional unibalanced unimodular pivotal Hopf algebra. Let $A$-mod be the category of finite dimensional modules over $A$. Let Proj be the ideal of projective objects in $A$-mod. The Hopf algebra $A$ itself with its left regular representation is a generator of Proj.

In Section 6 we prove the following theorem.

Theorem 2.5. There exist a non-degenerate $m$-trace on Proj in $A$-mod and a chromatic morphism $\widetilde{d}$ on the generator $A$ of Proj.

Let us briefly discuss the hypothesis on $A$. The existence of the chromatic morphism results from the theory of (co)integrals in Hopf algebras. The requirement that $A$ is pivotal implies that the category $A$-mod is pivotal. The unimodularity of $A$ ensures that the ideal Proj has a non-degenerate right $\mathrm{m}$-trace. That $A$ is unibalanced implies that this $\mathrm{m}$-trace is also a left m-trace, see 3 .

Theorems 2.4 and 2.5 yield an invariant of 3 -manifolds $\mathcal{K}_{A \text {-mod. }}$ By Lemmas 6.2 and 6.3 , the chromatic morphism $\tilde{d}$ is essentially determined by the integral $\lambda: A \rightarrow \mathbb{k}$ and cutting along a bounding circle is determined by the cointegral $\Lambda \in A$, where $\lambda(\Lambda)=1$.

In Section 6.3 we will prove the following theorem.

Theorem 2.6. If $A$ is involutive (i.e. the square of the antipode in $A$ is the identity map) then

$$
\mathcal{K}_{A-\bmod }(M)=\mathrm{Ku}_{A}(M)
$$

where $\mathrm{Ku}_{A}(M)$ is the Kuperberg invariant derived from A, see [16].

2.4. Turaev-Viro invariant. Let $\mathscr{C}$ be a finite semi-simple spherical $\mathbb{k}$ category and let $\left\{S_{i}\right\}_{i \in I}$ be a set of representatives of the isomorphism classes of simple objects of $\mathscr{C}$ (see [2]). The scalar

$$
\mathcal{D}=\sum_{i \in I} \operatorname{qdim}\left(S_{i}\right)^{2} \in \mathbb{k}
$$

is called the dimension of $\mathscr{C}$. We will explain that $\mathscr{C}$ satisfies the assumptions of Theorem 2.4, and, if $\mathcal{D} \neq 0$, then the resulting 3 -manifold invariant $\mathcal{K}_{\mathscr{C}}$ is equal to the Turaev-Viro invariant $\mathrm{TV}_{\mathscr{C}}$ [28, 1] associated to $\mathscr{C}$.

Observe that here $G=\oplus_{i \in I} S_{i}$ is a generator of $\mathcal{I}=\mathscr{C}$ and the quantum trace $\mathrm{t}=\mathrm{q}^{\operatorname{Tr}_{\mathscr{C}}}$ is a non-degenerate $\mathrm{m}$-trace on $\mathscr{C}$. It follows that

$$
\left\{x_{i}=\frac{1}{\mathrm{qdim}\left(S_{i}\right)}{\stackrel{\operatorname{cov}}{S_{i}}}\right\}_{i \in I} \text { and }\left\{y_{i}=\overrightarrow{\mathrm{ev}}_{S_{i}}\right\}_{i \in I}
$$


are dual bases of $\operatorname{Hom}_{\mathscr{C}}\left(\mathbb{1}, G \otimes G^{*}\right)$ and $\operatorname{Hom}_{\mathscr{C}}\left(G \otimes G^{*}, \mathbb{1}\right)$, respectively. Using the expansion $\Omega_{G \otimes G^{*}}=\sum_{i \in I} x_{i} \otimes_{\mathbb{k}} y_{i}$, it is straightforward to check that

$$
\widetilde{d}=\sum_{i \in I} \operatorname{qdim}\left(S_{i}\right) \operatorname{Id}_{S_{i}} \otimes \operatorname{Id}_{G}
$$

is a chromatic morphism for $G$. In Section 7 we prove the following theorem.

Theorem 2.7. If $\mathscr{C}$ is a finite semisimple spherical $\mathbb{k}$-category of non-zero dimension $\mathcal{D}$ with chromatic morphism $\widetilde{d}$ and generator $G$ then the invariant $\mathcal{K}_{\mathscr{C}}$ is proportional to the Turaev-Viro invariant of 3-manifolds associated to $\mathscr{C}$ :

$$
\mathrm{TV}_{\mathscr{C}}=\mathcal{D}^{-1} \mathcal{K}_{\mathscr{C}}
$$

2.5. Open Problems. Besides the categories discussed here, our approach certainly applies in other settings. Here we list (from least to most general) three further categories where our constructions should work:

(1) the categories of finite dimensional modules over nice (quantum) Lie super algebras, see [22, 23],

(2) the categories of finite dimensional modules over nice quasi-Hopf algebras, see [5, 6, 18, 20],

(3) general unimodular finite tensor categories, see [24].

Here the adjective "nice" means that the category satisfies the hypothesis of Theorem 2.3, in particular, admits an m-trace and a chromatic morphism. The theory of [12] should imply the existence of an m-trace in the above contexts though we may need to choose an appropriate pivotal structure to make the m-trace two sided, and it may be useful to work with the ideal of projective modules. It seems likely that the references listed above can help to construct chromatic morphisms in the categories in question. It is plausible that the results of [11] may help to generalize our approach to non-unimodular categories.

In a different direction, recall that Kuperberg [17] used framings of 3manifolds to generalize the invariant in [16] to arbitrary finite dimensional Hopf algebras. As explained above, a finite dimensional unibalanced unimodular pivotal Hopf algebra $A$ gives rise to a framing-independent 3-manifold invariant $\mathcal{K}_{A \text {-mod }}$ which is computed in a way similar to the invariants in [17] using an integral and a cointegral (see Section 6). With Theorem 2.6 in mind, we ask if $\mathcal{K}_{A \text {-mod }}(M)=\mathrm{Ku}_{A}(M, f)$ for some framing $f$ of a 3-manifold $M$ ? Is the Kuperberg invariant associated to a unibalanced unimodular pivotal Hopf algebra framing-independent? (This is known not be true for all finite dimensional Hopf algebras.)

\section{The AlgebraiC SETUP}


3.1. Pivotal and ribbon categories. In this paper, we consider strict tensor categories with tensor product $\otimes$ and unit object $\mathbb{1}$. Let $\mathscr{C}$ be such a category. The notation $V \in \mathscr{C}$ means that $V$ is an object of $\mathscr{C}$.

The category $\mathscr{C}$ is a pivotal category if it has duality morphisms

$$
\begin{array}{ll}
\overleftarrow{c o v}_{V}: \mathbb{1} \rightarrow V \otimes V^{*}, & \overleftarrow{\mathrm{ev}}_{V}: V^{*} \otimes V \rightarrow \mathbb{1}, \\
\overrightarrow{\operatorname{coev}}_{V}: \mathbb{1} \rightarrow V^{*} \otimes V, & \overrightarrow{\mathrm{ev}}_{V}: V \otimes V^{*} \rightarrow \mathbb{1}
\end{array}
$$

which satisfy compatibility conditions (see for example [2, 11]).

3.2. $\mathbb{k}$-categories. Let $\mathbb{k}$ be a field. A $\mathbb{k}$-category is a category $\mathscr{C}$ such that its hom-sets are left $\mathbb{k}$-modules, the composition of morphisms is $\mathbb{k}$-bilinear, and the canonical $\mathbb{k}$-algebra map $\mathbb{k} \rightarrow \operatorname{End}_{\mathscr{C}}(\mathbb{1}), k \mapsto k \operatorname{Id}_{\mathbb{1}}$ is an isomorphism. A tensor $\mathbb{k}$-category is a tensor category $\mathscr{C}$ such that $\mathscr{C}$ is a $\mathbb{k}$-category and the tensor product of morphisms is $\mathbb{k}$-bilinear.

3.3. M-traces on ideals in pivotal categories. Let $\mathscr{C}$ be a pivotal $\mathbb{k}$ category. Here we recall the definition of an m-trace on an ideal in $\mathscr{C}$, for more details see [11, 14]. By an ideal of $\mathscr{C}$ we mean a full subcategory, $\mathcal{I}$, of $\mathscr{C}$ which is

(1) Closed under $\otimes:$ If $V \in \mathcal{I}$ and $W \in \mathscr{C}$, then $V \otimes W$ and $W \otimes V$ are objects of $\mathcal{I}$.

(2) Closed under retractions: If $V \in \mathcal{I}, W \in \mathscr{C}$ and there are morphisms $f: W \rightarrow V, g: V \rightarrow W$ such that $g f=\operatorname{Id}_{W}$, then $W \in \mathcal{I}$.

An $m$-trace on an ideal $\mathcal{I}$ is a family of linear functions

$$
\left\{\mathrm{t}_{V}: \operatorname{End}_{\mathscr{C}}(V) \rightarrow \mathbb{k}\right\}_{V \in \mathcal{I}}
$$

such that following two conditions hold:

(1) Cyclicity: If $U, V \in \mathcal{I}$ then for any morphisms $f: V \rightarrow U$ and $g: U \rightarrow V$ in $\mathscr{C}$ we have $\mathrm{t}_{V}(g f)=\mathrm{t}_{U}(f g)$.

(2) Partial trace properties: If $U \in \mathcal{I}$ and $W \in \mathscr{C}$ then for any $f \in \operatorname{End}_{\mathscr{C}}(U \otimes W)$ and $g \in \operatorname{End}_{\mathscr{C}}(W \otimes U)$ we have

$$
\begin{gathered}
\mathrm{t}_{U \otimes W}(f)=\mathrm{t}_{U}\left(\left(\operatorname{Id}_{U} \otimes \overrightarrow{\mathrm{ev}}_{W}\right)\left(f \otimes \operatorname{Id}_{W^{*}}\right)\left(\operatorname{Id}_{U} \otimes \overleftarrow{c o e v}_{W}\right)\right) \\
\mathrm{t}_{W \otimes U}(g)=\mathrm{t}_{U}\left(\left(\overleftarrow{\mathrm{ev}}_{W} \otimes \operatorname{Id}_{U}\right)\left(\operatorname{Id}_{W^{*}} \otimes g\right)\left(\overrightarrow{\operatorname{cov}}_{W} \otimes \operatorname{Id}_{U}\right)\right)
\end{gathered}
$$

An $m$-trace on $\mathcal{I}$ is non-degenerate if for any $P \in \mathcal{I}$, the following pairing is non-degenerate:

$$
\operatorname{Hom}_{\mathscr{C}}(\mathbb{1}, P) \times \operatorname{Hom}_{\mathscr{C}}(P, \mathbb{1}) \rightarrow \mathbb{k},(x, y) \mapsto \mathrm{t}_{P}(x y) .
$$

Using the pivotal structure and the partial trace property one can deduce from the non-degeneracy condition that for all $P \in \mathcal{I}$ and $V \in \mathscr{C}$, the pairing

$$
\operatorname{Hom}_{\mathscr{C}}(V, P) \times \operatorname{Hom}_{\mathscr{C}}(P, V) \rightarrow \mathbb{k},(x, y) \mapsto \mathrm{t}_{P}(x y)
$$


is non-degenerate.

3.4. Projective objects. An object $P$ of $\mathscr{C}$ is projective if for any epimorphism $p: X \rightarrow Y$ and any morphism $f: P \rightarrow Y$ in $\mathscr{C}$, there exists a morphism $g: P \rightarrow X$ in $\mathscr{C}$ such that $f=p g$. An object $Q$ of $\mathscr{C}$ is injective if for any monomorphism $i: X \rightarrow Y$ and any morphism $f: X \rightarrow Q$ in $\mathscr{C}$, there exists a morphism $g: Y \rightarrow Q$ in $\mathscr{C}$ such that $f=g i$. Denote by Proj the full subcategory of projective objects. In a pivotal category projective and injective objects coincide (see [14]). Also, Proj is an ideal.

3.5. Invariants of colored ribbon graphs. Let $\mathscr{C}$ be a pivotal $\mathbb{k}$-category. A morphism $f: V_{1} \otimes \cdots \otimes V_{n} \rightarrow W_{1} \otimes \cdots \otimes W_{m}$ in $\mathscr{C}$ can be represented by a box and arrows:

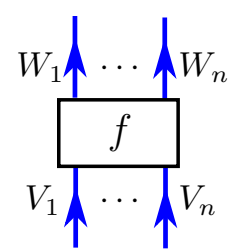

which are called coupons. All coupons have a top and a bottom sides which in our pictures will be the horizontal sides of the coupons. By a ribbon graph in an oriented manifold $\Sigma$, we mean an oriented compact surface embedded in $\Sigma$ which is decomposed into elementary pieces: bands, annuli, and coupons (see [26]) and is a thickening of an oriented graph. In particular, the vertices of the graph lying in $\operatorname{Int} \Sigma=\Sigma \backslash \partial \Sigma$ are thickened to coupons. A $\mathscr{C}$-colored ribbon graph is a ribbon graph whose (thickened) edges are colored by objects of $\mathscr{C}$ and whose coupons are colored by morphisms of $\mathscr{C}$. The intersection of a $\mathscr{C}$-colored ribbon graph in $\Sigma$ with $\partial \Sigma$ is required to be empty or to consist only of vertices of valency 1 . When $\Sigma$ is a surface the ribbon graph is just a tubular neighborhood of the graph.

A $\mathscr{C}$-colored ribbon graph in $\mathbb{R}^{2}$ (resp. $S^{2}=\mathbb{R}^{2} \cup\{\infty\}$ ) is called planar (resp. spherical). Let Rib be the category of planar $\mathscr{C}$-colored ribbon graphs and let $F: \operatorname{Rib} \rightarrow \mathscr{C}$ be the pivotal functor ${ }^{1}$ associated with $\mathscr{C}$ via the Penrose graphical calculus, see for example [14]. Let $\mathcal{L}_{a d m}$ be the class of all spherical $\mathscr{C}$-colored ribbon graphs obtained as the braid closure of a $(1,1)$ ribbon graph $T_{V}$ whose open edge is colored with an object $V \in \mathcal{I}$.

Given an m-trace $\mathrm{t}$ on $\mathcal{I}$ we can renormalize $F$ to an invariant

$$
F^{\prime}: \mathcal{L}_{a d m} \rightarrow \mathbb{k} \text { given by } F^{\prime}(L)=\mathrm{t}_{V}\left(F\left(T_{V}\right)\right)
$$

where $T_{V}$ is any $(1,1)$-ribbon graph as above. The properties of the m-trace imply that $F^{\prime}$ is a isotopy invariant of $L$, see [14].

\footnotetext{
${ }^{1}$ We call $F$ the pivotal functor because it can be associated with each pivotal category. Note, however, that the functor $F$ does not preserve the duality.
} 
Remark 3.1. If $\mathcal{I}$ is an ideal and $P \in \mathcal{I}$ then Lemma 2 of [14 implies that $P^{*} \in \mathcal{I}$. Moreover, the pivotal structure gives an isomorphism $f: P \rightarrow P^{* *}$ for all $P$. This isomorphism can be used to change the orientation of an edge of a graph as shown in the following diagram:

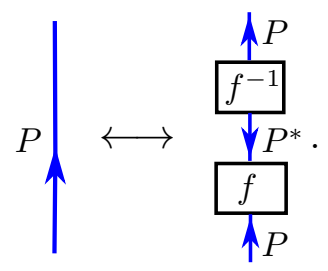

\section{Proof of Theorem 2.1}

4.1. Preliminaries. The following lemma contains standard facts from linear algebra; we leave the proof to the reader.

Lemma 4.1. Let $X_{j}$ and $Y_{j}$ be finite dimensional $\mathbb{k}$-modules, for $j=1,2$. Let $\langle,\rangle_{X_{j}, Y_{j}}: X_{j} \otimes_{\mathbb{k}} Y_{j} \rightarrow \mathbb{k}$ be a non-degenerate bilinear pairing (i.e. a pairing with trivial right and left kernels). Given a basis $\left\{x_{i}^{j}\right\}$ of $X_{j}$ let $\left\{y_{i}^{j}\right\}$ be the dual basis of $Y_{j}$ determined by $\left\langle x_{i}^{j}, y_{j}^{j}\right\rangle_{X_{j}, Y_{j}}=\delta_{i, j}$. Then

(1) the element $\Omega_{j}=\sum_{i} x_{i}^{j} \otimes_{\mathbb{k}} y_{i}^{j} \in X_{j} \otimes Y_{j}$ is independent of the choice of the basis $\left\{x_{i}^{j}\right\}$,

(2) if $h: X_{1} \rightarrow X_{2}$ and $k: Y_{2} \rightarrow Y_{1}$ are $\mathbb{k}$-linear maps such that $\langle h(x), y\rangle_{X_{2}, Y_{2}}=\langle x, k(y)\rangle_{X_{1}, Y_{1}}$ for all $x \in X_{1}$ and $y \in Y_{2}$ then

$$
\left(h \otimes \operatorname{Id}_{Y_{1}}\right)\left(\Omega_{1}\right)=\left(\operatorname{Id}_{X_{2}} \otimes k\right)\left(\Omega_{2}\right) .
$$

Proposition 4.2. Let $P \in \mathcal{I}$ and $\Omega_{P}=\sum_{i} x_{i} \otimes_{\mathbb{k}} y_{i}$ as in Formula (1). Let $\Lambda_{P}=\sum_{i} x_{i} y_{i} \in \operatorname{End}_{\mathscr{C}}(P)$. Then

(1) $\Omega_{P}$ is independent of the choice of the basis of $\operatorname{Hom}_{\mathscr{C}}(\mathbb{1}, P)$;

(2) If $P^{\prime} \in \mathcal{I}$ and $\phi: P \rightarrow P^{\prime}$ is a morphism then

$$
\left(\phi \otimes_{\mathbb{k}} \operatorname{Id}_{\mathbb{1}}\right) \Omega_{P}=\Omega_{P^{\prime}}\left(\operatorname{Id}_{\mathbb{1}} \otimes_{\mathbb{k}} \phi\right) \text { and } \phi \circ \Lambda_{P}=\Lambda_{P^{\prime}} \circ \phi ;
$$

(3) For any morphisms $f: \mathbb{1} \rightarrow P$ and $g: P \rightarrow \mathbb{1}$, we have

$$
\mathrm{t}_{P}(f g)=\sum_{i} \mathrm{t}_{P}\left(f y_{i}\right) \mathrm{t}_{P}\left(x_{i} g\right) \text {. }
$$

Proof. Set

$$
\begin{array}{ll}
X_{1}=\operatorname{Hom}_{\mathscr{C}}(\mathbb{1}, P), & Y_{1}=\operatorname{Hom}_{\mathscr{C}}(P, \mathbb{1}), \\
X_{2}=\operatorname{Hom}_{\mathscr{C}}\left(\mathbb{1}, P^{\prime}\right), & Y_{2}=\operatorname{Hom}_{\mathscr{C}}\left(P^{\prime}, \mathbb{1}\right) .
\end{array}
$$

For $j=1,2$, define the bilinear pairing $\langle,\rangle_{X_{j}, Y_{j}}: X_{j} \otimes Y_{j} \rightarrow \mathbb{k}$ by

$$
\langle x, y\rangle_{X_{1}, Y_{1}}=\mathrm{t}_{P}(x \circ y), \text { resp. }\langle x, y\rangle_{X_{2}, Y_{2}}=\mathrm{t}_{P^{\prime}}(x \circ y)
$$


for $x \in X_{j}$ and $y \in Y_{j}$. Clearly, Claim (1) of the proposition is a direct consequence of Claim (1) of Lemma 4.1. Next, we apply Claim (2) of the lemma to the linear maps

$$
\begin{gathered}
h: \operatorname{Hom}_{\mathscr{C}}(\mathbb{1}, P) \rightarrow \operatorname{Hom}_{\mathscr{C}}\left(\mathbb{1}, P^{\prime}\right) \text { given by } f \mapsto \phi \circ f, \\
k: \operatorname{Hom}_{\mathscr{C}}\left(P^{\prime}, \mathbb{1}\right) \rightarrow \operatorname{Hom}_{\mathscr{C}}(P, \mathbb{1}) \text { given by } g \mapsto g \circ \phi .
\end{gathered}
$$

Then Formula (9) yields the first equality in (10). Writing this equality explicitly we get

$$
\sum_{i}\left(\phi \circ x_{i}\right) \otimes y_{i}=\sum_{i} x_{i}^{\prime} \otimes\left(y_{i}^{\prime} \circ \phi\right)
$$

where $\Omega_{P^{\prime}}=\sum_{i} x_{i}^{\prime} \otimes_{\mathbb{k}} y_{i}^{\prime}$ and $\Lambda_{P^{\prime}}=\sum_{i} x_{i}^{\prime} y_{i}^{\prime} \in \operatorname{End}_{\mathscr{C}}\left(P^{\prime}\right)$ and the second equality in 10 follows.

To prove Claim (3) of the proposition, expand $f=\sum a_{i} x_{i}$ and $g=\sum b_{i} y_{i}$ where $a_{i}, b_{i} \in \mathbb{k},\left\{x_{i}\right\}$ is a basis of $X_{1}=\operatorname{Hom}_{\mathscr{C}}(\mathbb{1}, P)$ and $\left\{y_{i}\right\}$ is the dual basis of $Y_{1}=\operatorname{Hom}_{\mathscr{C}}(P, \mathbb{1})$ determined by the pairing $\langle,\rangle_{X_{1}, Y_{1}}$. Then

$$
\mathrm{t}_{P}(f g)=\sum_{i, j} a_{i} b_{j} \mathrm{t}_{P}\left(x_{i} y_{j}\right)=\sum_{i} a_{i} b_{i} .
$$

We also have $\mathrm{t}_{P}\left(f y_{i}\right)=\sum_{j} a_{j} \mathrm{t}_{P}\left(x_{j} y_{i}\right)=a_{i}$ and similarly $\mathrm{t}_{P}\left(x_{i} g\right)=b_{i}$ so Claim (3) follows.

4.2. Proof of Theorem 2.1. By the genus of a multi-handlebody we mean the sum of the genera of its components. We proceed by induction on the genus. The induction base concerns graphs on disjoint unions of 3-balls. Conditions (1) and (2) in Theorem 2.1 define $F^{\prime}$ uniquely for $\mathcal{I}$-colored graphs on the boundary of a topological 3-ball. Condition (3) extends $F^{\prime}$ uniquely to $\mathcal{I}$-colored graphs on disjoint unions of 3-balls. Note that cutting a 3-ball along a 2-disc as in Condition (4) produces two 3-balls. To conclude the argument in the base case we check Condition (4) for an arbitrary $\mathcal{I}$-colored graph $\left(B^{3}, \Gamma\right) \in \mathscr{H}_{\mathcal{I}}$ on the boundary of a 3 -ball $B^{3}$.

Let $D \subset B^{3}$ be an oriented properly embedded disc whose boundary intersects $\Gamma$ transversely (at the edges). Deforming if necessary $D$, we can view the circle $\partial D$ as the equator of the 2 -sphere $\partial B^{3}$ transversely intersecting the edges of $\Gamma$ in several points. We provide these points with an order compatible with the cyclic order on the circle $\partial D$ whose orientation is induced by that of $D$. Let $P \in \mathcal{I}$ be the tensor product of the objects of $\mathcal{I}$ associated with the edges of $\Gamma$ traversing these points. Let $\Gamma_{l}$ and $\Gamma_{u}$ be the parts of $\Gamma$ lying in the lower and upper hemispheres, respectively (we use the orientation of $\partial D$ to distinguish them). Cutting $\left(B^{3}, \Gamma\right)$ along $D$, we obtain an $\mathcal{I}$-colored graph on a disjoint union of two 3-balls

$$
\left.\operatorname{cut}_{D}\left(B^{3}\right), \operatorname{cut}_{D}(\Gamma)\right)=\left(B^{3}, \Gamma_{l}^{\prime}\right) \sqcup\left(B^{3}, \Gamma_{u}^{\prime}\right)
$$


where the new graphs are obtained by closing $\Gamma_{l}$ and $\Gamma_{u}$ with coupons using the tensor $\Omega_{P}=\sum_{i} x_{i} \otimes_{\mathbb{k}} y_{i}$ as in Section 2. Consider the morphisms $F\left(\Gamma_{l}\right)$ : $\mathbb{1} \rightarrow P$ and $F\left(\Gamma_{u}\right): P \rightarrow \mathbb{1}$. By the definition of $F^{\prime}$ and Proposition 4.2, Claim (3), we have

$$
F^{\prime}\left(B^{3}, \Gamma\right)=\mathrm{t}_{P}\left(F\left(\Gamma_{l}\right) F\left(\Gamma_{u}\right)\right)=\sum_{i} \mathrm{t}_{P}\left(F\left(\Gamma_{l}\right) y_{i}\right) \mathrm{t}_{P}\left(x_{i} F\left(\Gamma_{u}\right)\right) .
$$

Since, by definition, $F^{\prime}\left(\Gamma_{l}^{\prime}\right)=\mathrm{t}_{P}\left(F\left(\Gamma_{l}\right) y_{i}\right)$ and $F^{\prime}\left(\Gamma_{u}^{\prime}\right)=\mathrm{t}_{P}\left(x_{i} F\left(\Gamma_{u}\right)\right)$, this computation verifies Condition $(4)$ for $\left(B^{3}, \Gamma\right)$ and $D$. This concludes the proof of the induction base.

Assume now that we have an integer $g \geq 1$ and an invariant $F^{\prime}$ defined for all colored ribbon graphs on multi-handlebodies of genus $<g$ and satisfying Conditions (1)-(4). Let $(H, \Gamma) \in \mathscr{H}_{\mathcal{I}}$ where $H$ is a multi-handlebody of genus $g$. Pick an oriented properly embedded disc $D \subset H$ such that $\partial D$ is an essential simple closed curve on $\partial H$ missing all the coupons and intersecting all the edges of $\Gamma$ transversely. Cutting $(H, \Gamma)$ along $D$ produces a multi-handlebody $\left(\operatorname{cut}_{D}(H), \operatorname{cut}_{D}(\Gamma)\right)$ of genus $g-1$ which we denote simply $\operatorname{cut}_{D}(\Gamma)$. Set $F^{\prime}(H, \Gamma)=F^{\prime}\left(\operatorname{cut}_{D}(\Gamma)\right)$. We claim that $F^{\prime}(H, \Gamma)$ is well defined, i.e. is independent of the choice of the disc $D$. Pick another oriented properly embedded disc $D^{\prime} \subset H$ as above. We will prove that $F^{\prime}\left(\operatorname{cut}_{D}(\Gamma)\right)=F^{\prime}\left(\operatorname{cut}_{D^{\prime}}(\Gamma)\right)$. Assume first $\partial D \cap \partial D^{\prime}=\emptyset$. Deforming, if necessary, $D^{\prime}$ in $H$, we can assume that $D \cap D^{\prime}=\emptyset$. Then

$$
F^{\prime}\left(\operatorname{cut}_{D^{\prime}}(\Gamma)\right)=F^{\prime}\left(\operatorname{cut}_{D}\left(\operatorname{cut}_{D^{\prime}}(\Gamma)\right)\right)=F^{\prime}\left(\operatorname{cut}_{D^{\prime}}\left(\operatorname{cut}_{D}(\Gamma)\right)\right)=F^{\prime}\left(\operatorname{cut}_{D}(\Gamma)\right)
$$

where the first and the last equalities hold because, by the induction assumption, $F^{\prime}$ satisfies Condition (3) in genera $<g$. (This in particular proves that $F^{\prime}\left(\operatorname{cut}_{D}(\Gamma)\right)$ does not depend on the orientation of $D$ : choose $D^{\prime}$ to be parallel to $D$ but oriented in the opposite way.) If $\partial D \cap \partial D^{\prime} \neq \emptyset$, then it follows from [19], that there is a sequence $D_{0}=D, D_{1}, \ldots, D_{n}=D^{\prime}$ of oriented properly embedded discs in $H$ bounded by essential closed curves in $\partial H$ and such that $D_{i} \cap D_{i+1}=\emptyset$ for $i=0,1, \ldots, n-1$. By the above,

$$
F^{\prime}\left(\operatorname{cut}_{D_{0}}(\Gamma)\right)=F^{\prime}\left(\operatorname{cut}_{D_{1}}(\Gamma)\right)=\cdots=F^{\prime}\left(\operatorname{cut}_{D_{n}}(\Gamma)\right) .
$$

It is clear now that $F^{\prime}$ is defined for all colored ribbon graphs on multihandlebodies of genus $<g+1$ and satisfies Conditions (1)-(4). In particular, to check Condition (1), consider an orientation preserving diffeomorphism $f:(H, \Gamma) \rightarrow\left(H^{\prime}, \Gamma^{\prime}\right)$ of colored ribbon graphs on multi-handlebodies of genus $\leq g$. Pick a disc $D \subset H$ as above. By definition and induction assumption,

$$
F^{\prime}(H, \Gamma)=F^{\prime}\left(\operatorname{cut}_{D}(\Gamma)\right)=F^{\prime}\left(\operatorname{cut}_{f(D)}\left(\Gamma^{\prime}\right)\right)=F^{\prime}\left(H^{\prime}, \Gamma^{\prime}\right) .
$$

This completes the induction step. 


\section{INVARIANTS OF BICHROME GRAPHS AND 3-MANIFOLDS}

5.1. Bichrome handlebody graphs. For reader's convenience, we summarize here the terminology introduced above. A multi-handlebody is a disjoint union of a finite number of oriented 3-dimensional handlebodies. The genus of a multi-handlebody is the sum of the genera of its components. A bichrome handlebody graph is a pair $(H, \Gamma)$ where $H$ is a multi-handlebody and $\Gamma$ is a finite graph on $\partial H$ considered up to isotopy and formed by two disjoint subgraphs: $\Gamma=\Gamma_{\text {blue }} \sqcup \Gamma_{\text {red }}$ where $\Gamma_{\text {blue }} \subset \partial H$ is a $\mathscr{C}$-colored graph and $\Gamma_{\text {red }} \subset \partial H$ a union of unoriented disjoint simple closed curves called red circles. A bichrome handlebody graph $(H, \Gamma)$ is admissible if its blue subgraph is $\mathcal{I}$-colored and meets each connected component of $H$. We will sometimes drop $H$ and denote a bichrome handlebody graph $(H, \Gamma)$ simply by $\Gamma$. Finally, we let $\mathscr{H}_{b}$ denote the set of orientation preserving diffeomorphism classes of admissible bichrome handlebody graphs.

Definition 5.1. Let $H$ be a multi-handlebody.

(1) By a circle in $\partial H$ we mean an (unoriented) simple closed curve in $\partial H$.

(2) We say that a set of disjoint circles in $\partial H$ bounds in $H$ if these circles bound a disjoint union of discs embedded in $H$.

(3) A bounding set of circles in $\partial H$ is a reducing set if the complement of these circles in $\partial H$ is a disjoint union of 2 -spheres with holes.

(4) The complexity of a circle $\gamma$ in $\partial H$ is the minimal number of intersections of $\gamma$ with a reducing set of circles.

Definition 5.2 (Red Capping and Digging moves). Let $(H, \Gamma)$ and $\left(H^{\prime}, \Gamma^{\prime}\right)$ be bichrome handlebody graphs. We say that $(H, \Gamma)$ is obtained from $\left(H^{\prime}, \Gamma^{\prime}\right)$ by a red capping move along a red circle $c \subset \Gamma_{\text {red }}^{\prime}$ if

(1) there is a properly embedded disc $D \subset H^{\prime}$ such that the circle $\partial D \subset$ $\partial H^{\prime}$ transversely intersects $c$ in one point,

(2) $H$ is obtained from $H^{\prime}$ by attaching a 2-handle along $c$, and

(3) $\Gamma=\Gamma^{\prime} \backslash c$, where we identify the complement in $\partial H^{\prime}$ of an annulus neighborhood of $c$ with a subset of $\partial H$.

Conversely, we say $\left(H^{\prime}, \Gamma^{\prime}\right)$ is obtained from $(H, \Gamma)$ by a red digging move (producing the red circle $c$ ), see Figure 2.

Proposition 5.3. A red circle in a bichrome handlebody graph is the result of a red digging move if and only if it has complexity one.

Proof. Let $c$ be a red circle in a bichrome handlebody graph $\left(H^{\prime}, \Gamma^{\prime}\right)$. If $c$ has complexity one then there is a reducing set $S$ of circles in $\partial H^{\prime}$ such that $c \cap S=\{p t\}$. Attaching a 2-handle to $H^{\prime}$ along $c$, we get a multihandlebody $H$, and the graph $\left(H, \Gamma^{\prime} \backslash c\right)$ is obtained from $\left(H^{\prime}, \Gamma^{\prime}\right)$ by a red 


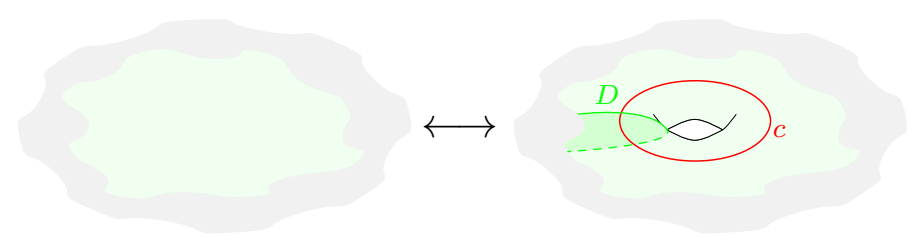

Figure 2. Red Digging / Capping moves

capping move. Its inverse is the required digging move. Conversely, if $c$ is obtained by a red digging move then it intersects exactly once the circle $\partial D$ in Definition 5.2 .

Proposition 5.4. Let $(H, \Gamma),\left(H, \Gamma^{\prime}\right) \in \mathscr{H}_{b}$ be bichrome graphs such that $\Gamma^{\prime}$ is obtained from $\Gamma$ by sliding a blue or red edge of $\Gamma$ over one of its red circles. Then $\Gamma$ and $\Gamma^{\prime}$ are related by a sequence of red digging and capping moves.

Proof. Let $\Gamma=\Gamma_{\text {blue }} \cup \Gamma_{\text {red }}$ and let $c \subset \Gamma_{\text {red }}$ be a red circle on which we want to slide an edge $e$ of $\Gamma$. Claim: up to applying one red digging move which transforms $(H, \Gamma)$ into a new bichrome graph $\left(H_{1}, \Gamma_{1}\right)$ and creates a new red circle $c^{\prime} \subset \Gamma_{1}$, we can reduce ourselves to the case where $c$ is a red circle created by a red digging move. This claim would imply the proposition because after applying a red capping move on $c$, we get a bichrome graph $\left(H_{2}, \Gamma_{2}\right)$ in which the edge $e$ can be slid, by an isotopy, over the disc added by the red capping. Re-digging along the same disc and re-capping along $c^{\prime}$, we get the bichrome graph $\left(H, \Gamma^{\prime}\right)$ obtained by sliding $e$ over $c$.

The claim above is proved by describing a suitable red digging move. Let $I \subset \partial H$ be a parametrized segment, i.e. the image of an embedding $i$ : $[-1,1] \hookrightarrow \partial H$ such that $\partial I \cap \Gamma=i(\{0\})$ is formed by a single point belonging to $c$. Let $I^{\prime}$ be a properly embedded arc in $H$ obtained by slightly pushing $I$ inside $H$ (keeping $\partial I$ fixed). Also, set

$$
H_{1}=H \backslash T u b\left(I^{\prime}\right), \quad c^{\prime}=\partial T u b(i(1)), \quad\left(\Gamma_{1}\right)_{b l u e}=\Gamma_{\text {blue }}, \quad\left(\Gamma_{1}\right)_{\text {red }}=\Gamma_{\text {red }} \sqcup c^{\prime}
$$

where $T u b$ stands for a tubular neighborhood and we view $\partial H \backslash T u b(\{i( \pm 1)\})$ as a subset of $\partial H_{1}$. Then $\left(H_{1}, \Gamma_{1}\right)$ is obtained from $(H, \Gamma)$ by a red digging move along $I$ which creates the red circle $c^{\prime}$. But, in the notation of Definition 5.2, $c$ intersects the disc $D$ bounded by $I \cup I^{\prime}$ exactly once so it has complexity 1. By Proposition 5.3, $c$ is created by a red digging move.

5.2. Heegaard splittings. A Heegaard splitting for a closed connected oriented 3-manifold $M$ is an ordered triple $\left(H_{\alpha}, H_{\beta}, \Sigma\right)$ where: (1) $H_{\alpha}$ and $H_{\beta}$ are handlebodies embedded in $M$ and endowed with orientation induced from that of $M$, (2) $H_{\alpha} \cap H_{\beta}=\partial H_{\alpha}=\partial H_{\beta}$; (3) the surface $\Sigma=\partial H_{\alpha}=\partial H_{\beta}$ is oriented as the boundary of $H_{\alpha}$ (with the outgoing vector first convention). 
Note that under these assumptions we have $H_{\alpha} \cup H_{\beta}=M$. A Heegaard diagram of $M$ compatible with the Heegaard splitting $\left(H_{\alpha}, H_{\beta}, \Sigma\right)$ is a triple $\left(\Sigma,\left\{\alpha_{i}\right\},\left\{\beta_{i}\right\}\right)$ where $\left\{\alpha_{i}\right\}$ and $\left\{\beta_{i}\right\}$ are minimal reducing sets of circles in $\Sigma$ bounding in $H_{\alpha}$ and $H_{\beta}$, respectively. Clearly, from the Heegaard diagram one can recover $H_{\alpha}, H_{\beta}$, and $M=H_{\alpha} \cup_{\Sigma} H_{\beta}$ up to diffeomorphism.

By Section 2.2, a Heegaard diagram $\left(\Sigma,\left\{\alpha_{i}\right\},\left\{\beta_{i}\right\}\right)$ of $M$ determines a bichrome diagram for $M$ represented by the bichrome handlebody graph $\left(H_{\alpha}, \Gamma_{\text {blue }} \cup \Gamma_{\text {red }}\right)$ where $\Gamma_{\text {red }}=\left\{\beta_{i}\right\}$ and $\Gamma_{\text {blue }}$ is the planar ribbon graph $\mathbf{O}_{G}$ which is the braid closure of the coupon filled with $h$ such that $\mathrm{t}_{G}(h)=1$. (Since $H_{\alpha} \backslash \Gamma_{r e d}$ is connected, the position of $\mathbf{O}_{G}$ is unique up to isotopy.)

Theorem 5.5. Any two bichrome diagrams for $M$ are related by a finite sequence of red digging and capping moves.

Proof. Let $(H, \Gamma)$ and $\left(H^{\prime}, \Gamma^{\prime}\right)$ be bichrome diagrams for $M$. It is well known that $\left(H, \Gamma_{r e d}\right)$ and $\left(H^{\prime}, \Gamma_{r e d}^{\prime}\right)$ can be related by a finite sequence of stabilization moves, the inverse moves, and handle slide moves (see [25]). A stabilization move is a special case of a red digging move (and its inverse is a red capping move). By Proposition 5.4, handle slides are compositions of isotopies with red digging and capping moves. All these moves can be applied here because $\Gamma_{\text {blue }}=\mathbf{O}_{G}$ lies in a disc in $\partial H$.

5.3. Proof of Theorems 2.3 and 2.4. We start with lemmas.

Lemma 5.6. Let $P, P^{\prime} \in \mathcal{I}$ and

$$
\Lambda_{P^{\prime} \otimes G^{*}}=\sum_{i} y_{i} x_{i} \in \operatorname{End}_{\mathscr{C}}\left(P^{\prime} \otimes G^{*}\right) \text { where } \Omega_{P^{\prime} \otimes G^{*}}=\sum x_{i} \otimes_{\mathbb{k}} y_{i}
$$

Then

$$
\left(\operatorname{Id}_{P^{\prime}} \otimes \overleftarrow{\mathrm{ev}}_{G} \otimes \operatorname{Id}_{P}\right) \circ\left(\Lambda_{P^{\prime} \otimes G^{*}} \otimes \widetilde{d}_{P}\right) \circ\left(\operatorname{Id}_{P^{\prime}} \otimes \overrightarrow{\operatorname{cov}}_{G} \otimes \operatorname{Id}_{P}\right)=\operatorname{Id}_{P^{\prime} \otimes P}
$$

Proof. Since $G$ is a generator of $\mathcal{I}$ there exist $g_{i}: P \rightarrow G, f_{i}: G \rightarrow P$ and $g_{i}^{\prime}: P^{\prime} \rightarrow G, f_{i}^{\prime}: G \rightarrow P^{\prime}$ such that $\sum_{i} f_{i} \circ g_{i}=\operatorname{Id}_{P}$ and $\sum_{i} f_{i}^{\prime} \circ g_{i}^{\prime}=\operatorname{Id}_{P^{\prime}}$. 
Then

$$
\begin{aligned}
\operatorname{Id}_{P^{\prime} \otimes P} & =\sum_{i, j}\left(f_{i}^{\prime} \otimes f_{j}\right) \circ \operatorname{Id}_{G \otimes G} \circ\left(g_{i}^{\prime} \otimes g_{j}\right) \\
& =\sum_{i, j}\left(f_{i}^{\prime} \otimes \overleftarrow{\mathrm{ev}}_{G} \otimes f_{j}\right) \circ(\Lambda \otimes \widetilde{d}) \circ\left(g_{i}^{\prime} \otimes \overrightarrow{\operatorname{cov}}_{G} \otimes g_{j}\right) \\
& =\sum_{i}\left(f_{i}^{\prime} \otimes \overleftarrow{\mathrm{ev}}_{G} \otimes \operatorname{Id}_{P}\right) \circ\left(\Lambda \otimes \widetilde{d}_{P}\right) \circ\left(g_{i}^{\prime} \otimes \overrightarrow{\mathrm{cev}}_{G} \otimes \operatorname{Id}_{P}\right) \\
& \left.=\sum_{i}\left(\operatorname{Id}_{P^{\prime}} \otimes \overleftarrow{\mathrm{ev}}_{G} \otimes \operatorname{Id}_{P}\right) \circ\left(\left(f_{i}^{\prime} \otimes \operatorname{Id}_{G^{*}}\right) \Lambda\left(g_{i}^{\prime} \otimes \operatorname{Id}_{G^{*}}\right)\right) \otimes \widetilde{d}_{P}\right) \circ \\
& =\sum_{i}\left(\operatorname{Id}_{P^{\prime}} \otimes \overleftarrow{\mathrm{ev}}_{G} \otimes \operatorname{Id}_{P}\right) \circ\left(\left(\Lambda_{P^{\prime}} \otimes G^{*} \circ\left(f_{i}^{\prime} g_{i}^{\prime} \otimes \operatorname{Id}_{G^{*}}\right)\right) \otimes \widetilde{d}_{P}\right) \circ \\
& \circ\left(\operatorname{Id}_{P^{\prime}} \otimes \overrightarrow{\operatorname{covv}}_{G} \otimes \operatorname{Id}_{P}\right) \\
& =\sum_{i}\left(\operatorname{Id}_{P^{\prime}} \otimes \overleftarrow{\mathrm{ev}}_{G} \otimes \operatorname{Id}_{P}\right) \circ\left(\Lambda_{P^{\prime} \otimes G^{*}} \otimes \widetilde{d}_{P}\right) \circ\left(\operatorname{Id}_{P^{\prime}} \otimes \overrightarrow{\operatorname{cev}}_{G} \otimes \operatorname{Id}_{P}\right)
\end{aligned}
$$

where the second equality uses the definition of the chromatic morphism (Formula (3)), the third equality uses the definition of $\widetilde{d}_{P}$ (Formula (50) and the second to last equality comes from Part (2) of Proposition 4.2 where $\phi=f_{i}^{\prime} \otimes \operatorname{Id}_{G^{*}}$.

Given a bichrome handlebody graph $(H, \Gamma)$ we can produce a new bichrome handlebody graph $\left(H^{\prime}, \Gamma^{\prime}\right)$ by doing a red digging move on $(H, \Gamma)$ then changing the newly created red circle into a blue graph using the chromatic morphism, as in Formula (6). We say $\left(H^{\prime}, \Gamma^{\prime}\right)$ is obtained from $(H, \Gamma)$ by a blue digging move. Conversely, we say $(H, \Gamma)$ is obtained from $\left(H^{\prime}, \Gamma^{\prime}\right)$ by a blue capping move. See Figure 3 for a pictorial representation of these moves.

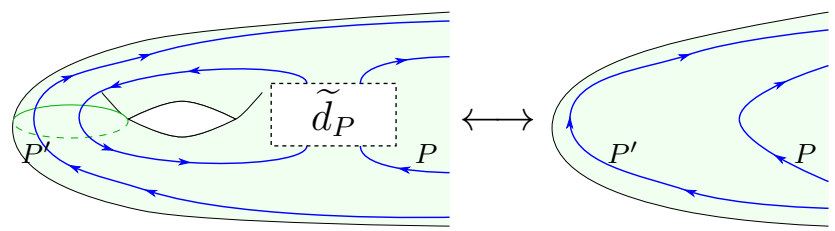

Figure 3. Blue Digging and Capping Moves. Here one can assume the orientation of the leftmost strand is as shown in the figure, cf. Remark 3.1.

Lemma 5.7. The invariant $F^{\prime}$ of $\mathcal{I}$-colored ribbon graphs on multi-handlebodies defined in Theorem 2.1 is preserved under blue digging and capping moves. 
Proof. Let $(H, \Gamma)$ and $\left(H^{\prime}, \Gamma^{\prime}\right)$ be bichrome handlebody graphs such that $\left(H^{\prime}, \Gamma^{\prime}\right)$ is obtained from $(H, \Gamma)$ by a blue digging move. Up to cutting along a reducing set of curves for $H$, we can assume that the component of $H$ to which we are applying the blue digging has genus 0 . To prove the lemma we compute the morphisms associated to the subsurfaces of $\partial H$ and $\partial H^{\prime}$ drawn in Figure 3. Clearly, the morphism associated with the right hand side is $\operatorname{Id}_{P^{\prime}} \otimes \operatorname{Id}_{P}$. To compute the morphism, say $f$, appearing on the left hand side, let $\gamma$ be the curve bounding the disc in the far left part of Figure 3 . Cutting along $\gamma$, as discussed before Theorem 2.1, we get that

$$
f=\left(\operatorname{Id}_{P^{\prime}} \otimes \overleftarrow{\mathrm{ev}}_{G} \otimes \operatorname{Id}_{P}\right) \circ\left(\Lambda_{P^{\prime} \otimes G^{*}} \otimes \widetilde{d}_{P}\right) \circ\left(\operatorname{Id}_{P^{\prime}} \otimes \overrightarrow{\operatorname{cov}}_{G} \otimes \operatorname{Id}_{P}\right)
$$

By Lemma 5.6, $f=\operatorname{Id}_{P^{\prime}} \otimes \operatorname{Id}_{P}$.

Proof of Theorem 2.3. We prove the following slightly stronger claim.

Claim: There exists a unique extension of $F^{\prime}$ to an invariant of admissible bichrome handlebody graphs satisfying Formula (6) and invariant under the blue capping and digging moves.

We prove this by induction on the number of red circles. When there are no red circles the claim is just Lemma 5.7. Now suppose that the claim holds for all admissible bichrome handlebody graphs with $n-1$ red circles. Let $(H, \Gamma)$ be an admissible bichrome handlebody graph with $n$ red circles. Pick an injective path $\gamma_{0}$ in $\partial H \backslash \Gamma$ leading from a point on a blue edge to a point on a red circle $\beta_{0}$. Using $\gamma_{0}$, we pull a small segment of the blue edge to $\beta_{0}$ then use the chromatic morphism to change $\beta_{0}$ into a blue graph. We obtain an admissible bichrome handlebody graph $\left(H, \Gamma_{\gamma_{0}}\right)$ with $n-1$ red circles. By the induction assumption, $F^{\prime}\left(H, \Gamma_{\gamma_{0}}\right)$ is well defined. So, if $F^{\prime}$ exists, then it is unique. To prove that $F^{\prime}$ exists we show its independence of the choice of $\gamma_{0}$. Let $\gamma_{1}$ be another such path going from a point on a blue edge to a point on a red circle $\beta_{1}$ and let $\left(H, \Gamma_{\gamma_{1}}\right)$ be the admissible bichrome handlebody graph obtained as above using $\gamma_{1}$ to make $\beta_{1}$ blue. We separate two cases.

Case 1. $\beta_{0}=\beta_{1}$. Here we have two sub-cases. First, suppose the red circle $\beta_{0}=\beta_{1}$ has complexity one. By Proposition 5.3 , the circle $\beta_{0}$ is the result of a digging move. Therefore, when we use $\gamma_{0}$ or $\gamma_{1}$ and the chromatic morphism to change $\beta_{0}$ into a blue graph, we arrive at a diagram as in the left side of Figure 3. In both cases we can do a blue capping move to arrive at the same $\mathcal{I}$-colored ribbon graph on a multi-handlebody with $n-1$ red circles. Thus, induction implies that $F^{\prime}\left(H, \Gamma_{\gamma_{0}}\right)=F^{\prime}\left(H, \Gamma_{\gamma_{1}}\right)$. So, in this case the extension of $F^{\prime}$ does not depend on the choice of $\gamma_{0}$.

Second, assume the red curve $\beta_{0}=\beta_{1}$ has any complexity. Apply a blue digging move to $\Gamma$ along a small interval $I$ intersecting $\beta_{0}$ in a single point. The result is a new bichrome handlebody graph $\left(H^{\prime}, \Gamma^{\prime}\right)$ with $n$ red circles 
in which the image $\beta_{0}^{\prime}$ of $\beta_{0}$ is a red curve with complexity 1 . Since this digging move only modified $(H, \Gamma)$ in a neighborhood of $I$, we can identify $\gamma_{0}$ and $\gamma_{1}$ as paths in $\partial H^{\prime} \backslash \Gamma^{\prime}$ and we can apply the chromatic morphism to $\beta_{0}^{\prime}$ either through $\gamma_{0}$ and $\gamma_{1}$ getting, respectively, bichrome handlebody graphs $\Gamma_{\gamma_{0}}^{\prime}$ and $\Gamma_{\gamma_{1}}^{\prime}$ with $n-1$ red components. By the preceding case $F^{\prime}\left(H^{\prime}, \Gamma_{\gamma_{0}}^{\prime}\right)=$ $F^{\prime}\left(H^{\prime}, \Gamma_{\gamma_{1}}^{\prime}\right)$. Observing that $\Gamma_{\gamma_{0}}^{\prime}\left(\operatorname{resp} . \Gamma_{\gamma_{1}}^{\prime}\right)$ is obtained from $\Gamma_{\gamma_{0}}\left(\operatorname{resp} . \Gamma_{\gamma_{1}}\right)$ by a blue digging move, we get

$$
F^{\prime}\left(H, \Gamma_{\gamma_{0}}\right)=F^{\prime}\left(H^{\prime}, \Gamma_{\gamma_{0}}^{\prime}\right)=F^{\prime}\left(H^{\prime}, \Gamma_{\gamma_{1}}^{\prime}\right)=F^{\prime}\left(H, \Gamma_{\gamma_{1}}^{\prime}\right) \text {. }
$$

Case 2. $\beta_{0} \neq \beta_{1}$. We separate two sub-cases. First, suppose $\gamma_{0}$ and $\gamma_{1}$ are disjoint. In $\left(H, \Gamma_{\gamma_{0}}\right)$ (resp. $\left.\left(H, \Gamma_{\gamma_{1}}\right)\right)$ we can use $\gamma_{1}$ (resp. $\left.\gamma_{0}\right)$ to change $\beta_{1}$ (resp. $\beta_{0}$ ) into a blue graph and obtain an admissible bichrome handlebody graph $\left(H, \Gamma_{1}\right)$ with $n-2$ red circles. By the induction assumption,

$$
F^{\prime}\left(H, \Gamma_{\gamma_{0}}\right)=F^{\prime}\left(H, \Gamma_{1}\right)=F^{\prime}\left(H, \Gamma_{\gamma_{1}}\right) .
$$

Second, suppose $\gamma_{0} \cap \gamma_{1} \neq \emptyset$. We claim that there is another path $\gamma_{1}^{\prime}$ connecting $\Gamma_{\text {blue }}$ to $\beta_{1}$ such that $\gamma_{0} \cap \gamma_{1}^{\prime}=\emptyset$. Then by the previous subcase $F^{\prime}\left(H, \Gamma_{\gamma_{0}}\right)=F^{\prime}\left(H, \Gamma_{\gamma_{1}^{\prime}}\right)$ and by Case 1 we have $F^{\prime}\left(H, \Gamma_{\gamma_{1}^{\prime}}\right)=F^{\prime}\left(H, \Gamma_{\gamma_{1}}\right)$. So, the result follows. To prove our claim observe that since $\gamma_{0} \cap \gamma_{1} \neq \emptyset$. the paths $\gamma_{0}$ and $\gamma_{1}$ lie in the same connected component $R$ of $\partial H \backslash \Gamma$. Moreover, $R$ is an open orientable surface which is the interior of a compact surface with at least 3 distinct boundary components: $\partial_{\text {blue }} \subset \Gamma_{\text {blue }}, \beta_{0}$ and $\beta_{1}$. Then $\gamma_{0}$ and $\gamma_{1}$ are embedded arcs in $R$ connecting $\partial_{\text {blue }}$ to $\beta_{0}$ and $\beta_{1}$, respectively. But $R \backslash \gamma_{0}$ is connected as $\gamma_{0}$ intersects the closed curve $\beta_{0}$ once. Thus, there exists another path in $R \backslash \gamma_{0}$ connecting $\partial_{b l u e}$ and $\beta_{1}$.

Proof of Theorem 2.4. By Theorem 5.5 it is enough to show that $F^{\prime}$ is invariant under red digging and capping moves. Let $(H, \Gamma)$ and $\left(H^{\prime}, \Gamma^{\prime}\right)$ be bichrome handlebody graphs such that $\left(H^{\prime}, \Gamma^{\prime}\right)$ is obtained from $(H, \Gamma)$ by a red digging move. Suppose that $c$ is the red circle created by this move. Let $\left(H^{\prime}, \Gamma^{\prime \prime}\right)$ be the bichrome handlebody graph obtained from using an edge of the blue graph and the chromatic morphism to change the red circle $c$ into a blue graph. By definition, the composition of these two moves is a blue digging move. By Lemma 5.7 we have $F^{\prime}(H, \Gamma)=F^{\prime}\left(H^{\prime}, \Gamma^{\prime \prime}\right)$. Since $\left(H^{\prime}, \Gamma^{\prime}\right)$ and $\left(H^{\prime}, \Gamma^{\prime \prime}\right)$ differ by an isotopy and a move represented in Formula (6), Theorem 2.3 implies that $F^{\prime}\left(H^{\prime}, \Gamma^{\prime}\right)=F^{\prime}\left(H^{\prime}, \Gamma^{\prime \prime}\right)$. Combining these equalities we get $F^{\prime}(H, \Gamma)=F^{\prime}\left(H^{\prime}, \Gamma^{\prime}\right)$ which concludes the proof.

\section{Hopf Algebras and the Kuperberg invariants}

In this section we prove Theorems 2.5 and 2.6. First, we briefly recall some well known facts about Hopf algebras, see for example [21]. 
6.1. Hopf algebra preliminaries. Let $A$ be a finite-dimensional Hopf algebra over a field $\mathbb{k}$ with multiplication $m: A \otimes A \rightarrow A$, unit $\eta: \mathbb{k} \rightarrow A$, coproduct $\Delta: A \rightarrow A \otimes A$, counit $\epsilon: A \rightarrow \mathbb{k}$, antipode $S: A \rightarrow A$. A right integral of $A$ is a linear form $\lambda \in A^{*}$ such that $\lambda f=f\left(1_{A}\right) \cdot \lambda$ for all $f \in A^{*}$. This means that $\left(\lambda \otimes \operatorname{Id}_{A}\right)(\Delta(x))=\lambda(x) \cdot 1_{A}$ for every $x \in A$. A left (resp. right) cointegral of $A$ is a vector $\Lambda \in A$ satisfying $x \Lambda=\epsilon(x) \Lambda$ (resp. $\Lambda x=\epsilon(x) \Lambda)$ for all $x \in A$. Since $A$ is finite-dimensional, right integrals form a 1-dimensional ideal in $A^{*}$ and left cointegrals form a 1-dimensional ideal in $A$. Moreover, every non-zero right integral $\lambda \in A^{*}$ and every non-zero left cointegral $\Lambda \in A$ satisfy $\lambda(\Lambda) \neq 0$. We fix a right integral $\lambda \in A^{*}$ and a left cointegral $\Lambda \in A$ satisfying $\lambda(\Lambda)=1$. We will use sumless Sweedler's notation to describe the coproduct, for example, we write $\Delta^{3}(x)=x_{(1)} \otimes x_{(2)} \otimes x_{(3)}$.

The Hopf algebra $A$ is unimodular if $S(\Lambda)=\Lambda$, or equivalently, if $\Lambda$ is both a right and left cointegral. We call $A$ pivotal if there exists $g \in A$ such that $S^{2}(x)=g x g^{-1}$ for all $x \in A$. Let Proj be the ideal of projective modules over $A$ (for the definition of projective objects, see Section 3.4). By Theorem 1 of [3] every finite dimensional unimodular pivotal Hopf algebra has a left m-trace on Proj. Such a Hopf algebra is unibalanced if this left $\mathrm{m}$-trace is also a right $\mathrm{m}$-trace.

6.2. Proof of Theorem 2.5. Let $A$ be a finite dimensional unibalanced unimodular pivotal Hopf algebra over a field $\mathbb{k}$ and let $A$-mod be the category of its finite dimensional left modules. We denote by the same symbol $A$ the algebra $A$ viewed as a left $A$-module via the action $L: A \rightarrow \operatorname{End}_{\mathbb{k}}(A)$ given by $L_{h}(x)=h x$ for all $h, x \in A$.

Lemma 6.1. The left $A$-module $A$ is a generator of Proj in A-mod.

Proof. Let $P$ be an indecomposable projective $A$-module. Then $P$ is a direct summand of a direct sum of a finite number of copies of $A$. Since $P$ is indecomposable, $P$ is a direct summand of $A$. Since the Krull-Schmidt theorem holds in $A$-mod, every element of Proj is a direct sum of indecomposable projective modules and the lemma follows.

We let $\mathrm{t}$ be the non-degenerate $\mathrm{m}$-trace on Proj normalized so that $\mathrm{t}_{A}\left(f_{\Lambda} \circ\right.$ $\epsilon)=1$ where $f_{\Lambda}: \mathbb{k} \rightarrow A$ is the morphism carrying $1 \in \mathbb{k}$ to the left cointegral $\Lambda \in A$. For $P \in$ Proj, the map $\Lambda_{P}^{\prime}: P \rightarrow P$ determined by the left action of $\Lambda$ on $P$ is a morphism because $\Lambda$ is both a left and right cointegral. Consider also the morphism $\Lambda_{P}: P \rightarrow P$ given by $\Lambda_{P}=\sum_{i} x_{i} y_{i}$ where $\Omega_{P}=\sum_{i} x_{i} \otimes_{\mathbb{k}} y_{i}$ is defined by Formula (1).

Lemma 6.2. $\Lambda_{P}=\Lambda_{P}^{\prime}$.

Proof. First, consider the case when $P=A$. Since the space of cointegrals is one dimensional, the space $\operatorname{Hom}_{A-\bmod }(\mathbb{1}, A)$ also is one dimensional and 
is generated by the morphism $f_{\Lambda}: \mathbb{k} \rightarrow A$ defined above. Combining this with the fact that $\mathrm{t}$ is non-degenerate (with the normalization above) we get $\Omega_{A}=f_{\Lambda} \otimes_{\mathbb{k}} \epsilon$. Thus, for all $x \in A$ we have

$$
\Lambda_{A}(x)=f_{\Lambda} \epsilon(x)=\epsilon(x) \Lambda=x \Lambda=\Lambda_{A}^{\prime}(x) .
$$

So, the lemma holds for $P=A$.

Let $P$ be any object in Proj. Since $A$ is a generator of Proj in $A$-mod, there is a finite set of pairs of morphisms $\left\{f_{j}: A \rightarrow P, g_{j}: P \rightarrow A\right\}_{j \in J}$ such that $\operatorname{Id}_{P}=\sum_{j \in J} f_{j} g_{j}$. From Proposition 4.2, Part (2) we get

$$
\Omega_{P}=\sum_{j \in J}\left(\left(f_{j} \otimes \operatorname{Id}_{\mathbb{1}}\right) \Omega_{A}\left(\operatorname{Id}_{\mathbb{1}} \otimes g_{j}\right)\right)=\sum_{j \in J}\left(\left(f_{j} \circ f_{\Lambda}\right) \otimes\left(\epsilon \circ g_{j}\right)\right) .
$$

So by the definition of $\Lambda_{P}$, for $y \in P$, we have

$$
\begin{aligned}
\Lambda_{P}(y)=\sum_{j \in J}\left(f_{j} \circ f_{\Lambda} \circ \epsilon \circ g_{j}\right)(y) & =\sum_{j \in J} f_{j}\left(\epsilon\left(g_{j}(y)\right) \Lambda\right) \\
& =\sum_{j \in J} f_{j}\left(\Lambda g_{j}(y)\right) \\
& =\sum_{j \in J} \Lambda f_{j}\left(g_{j}(y)\right)=\Lambda y=\Lambda_{P}^{\prime}(y) .
\end{aligned}
$$

So, $\Lambda_{P}=\Lambda_{P}^{\prime}$.

In [8] it is shown that the right integral $\lambda \in A^{*}$ determines a morphism $f_{\lambda, 1_{A}}: A \rightarrow A^{*} \otimes A$ carrying $1_{A}$ to $\lambda \otimes 1_{A}$.

Lemma 6.3. The morphism $\tilde{d}: A \otimes A \rightarrow A \otimes A$ defined by

$$
\widetilde{d}=\left(\overrightarrow{\mathrm{ev}}_{A} \otimes \operatorname{Id}_{A \otimes A}\right)\left(\operatorname{Id}_{A} \otimes f_{\lambda, 1_{A}} \otimes \operatorname{Id}_{A}\right)\left(\operatorname{Id}_{A} \otimes \Delta\right)
$$

is a chromatic morphism for the generator $A$.

Proof. First, we set $h=\lambda\left(S\left(\Lambda_{(2)}\right)\right) \Lambda_{(1)}$ and prove that

$$
h=\lambda\left(S\left(\Lambda_{(2)}\right)\right) \Lambda_{(1)}=1_{A} .
$$

Observe that

$$
\begin{aligned}
S(h)=\left(\operatorname{Id}_{A} \otimes \lambda\right)(S \otimes S)(\Delta(\Lambda)) & =\left(\operatorname{Id}_{A} \otimes \lambda\right)\left(\Delta^{o p}(S(\Lambda))\right) \\
& =\left(\lambda \otimes \operatorname{Id}_{A}\right)(\Delta(S(\Lambda))) \\
& =\lambda(S(\Lambda)) 1_{A}=\lambda(\Lambda) 1_{A}=1_{A} .
\end{aligned}
$$

Since $A$ is finite dimensional, the antipode $S: A \rightarrow A$ is invertible and this computation implies Formula (12).

For $x \in X$, let $L_{x}$ be the left action of $x$ on $A$ or $A^{*}$. Note the identity

$$
\overleftarrow{\mathrm{ev}}_{A} \circ\left(\operatorname{Id}_{A^{*}} \otimes L_{x}\right) \circ f_{\lambda \otimes 1_{A}}=\lambda(x) \cdot \epsilon
$$


established in the proof of Lemma 3.8 of [8].

Let $g$ be the morphism on the left side of Formula (3). For any $x, y \in A$,

$$
\begin{aligned}
g(x \otimes y) & =\left(\operatorname{Id}_{A} \otimes \overleftarrow{\mathrm{ev}}_{A}\right)\left(\Lambda \otimes \operatorname{Id}_{A}\right)\left(\operatorname{Id}_{A} \otimes f_{\lambda \otimes 1_{A}} \otimes \operatorname{Id}_{A}\right)(x \otimes \Delta(y)) \\
& =\Lambda_{(1)} x \otimes\left(\overleftarrow{\mathrm{ev}}_{A}\left(L_{\Lambda_{(2)}} \otimes \operatorname{Id}_{A}\right) f_{\lambda \otimes 1_{A}}\left(y_{(1)}\right)\right) y_{(2)} \\
& =\Lambda_{(1)} x \otimes\left(\overleftarrow{\mathrm{ev}}_{A}\left(\operatorname{Id}_{A^{*}} \otimes L_{S\left(\Lambda_{(2)}\right)}\right) f_{\lambda \otimes 1_{A}}\left(y_{(1)}\right)\right) y_{(2)} \\
& =\Lambda_{(1)} x \otimes \lambda\left(S\left(\Lambda_{(2)}\right)\right) \epsilon\left(y_{(1)}\right) y_{(2)} \\
& =\lambda\left(S\left(\Lambda_{(2)}\right)\right) \Lambda_{(1)} x \otimes y \\
& =x \otimes y
\end{aligned}
$$

where the fourth equality follows from Formula (13), the fifth equality follows from the formula $\left(\epsilon \otimes \mathrm{Id}_{A}\right) \Delta=\operatorname{Id}_{A}$ in the definition of the Hopf algebra $A$, and the last equality follows from Formula (12).

Theorem 2.5 directly follows from the results of this subsection.

6.3. Proof of Theorem 2.6. In Section 6.2 we showed that the category $A$-mod satisfies the assumptions of Theorems 2.3 and 2.4. Thus we have:

Theorem 6.4. The category of finite dimensional modules over a finite dimensional unibalanced unimodular pivotal Hopf algebra A gives rise to an invariant of bichrome handlebody graphs $F^{\prime}$ and an invariant of 3-manifolds $\mathcal{K}_{A-\text { mod }}$.

We show how to compute $\mathcal{K}_{A \text {-mod }}(M)$ for a closed connected oriented 3manifold $M$. We will use the generator $G=A$ and choose $\mathbf{O}_{G}$ to be the ribbon graph formed by the braid closure of the coupon filled with $h=f_{\Lambda} \circ \epsilon$, see Section 2.2. Let $\left(H_{\alpha}, \Gamma\right)$ be a bichrome diagram determined by a Heegaard splitting $M=H_{\alpha} \cup_{\Sigma} H_{\beta}$ with lower and upper minimal reducing sets of circles $\left\{\alpha_{1}, \ldots, \alpha_{g}\right\}$ and $\left\{\beta_{1}, \ldots, \beta_{g}\right\}$, respectively. Using the blue graph $\mathbf{O}_{G}$ and the chromatic morphism we change all the red circles $\left\{\beta_{i}\right\}$ into a blue graph. Now $\left\{\alpha_{i}\right\}$ is a minimal reducing set of circles on $H_{\alpha}$ and so using Property (4) of Theorem 2.1 we can cut along the discs in $H_{\alpha}$ bounded by these circles to obtain a graph $\Gamma^{\prime}$ in the boundary of the 3-ball $B^{3}$. By definition,

$$
\mathcal{K}_{A-\bmod }(M)=F^{\prime}\left(B^{3}, \Gamma^{\prime}\right),
$$

see Figure 4 for an example.

In the rest of the section, we assume $A$ to be involutive so that $S^{2}=\mathrm{id}_{A}$ and compute $\mathcal{K}_{A \text {-mod }}(M)$. The involutivity of $A$ implies that we can choose the pivotal structure of $A$-mod to be trivial and then there is a forgetful pivotal functor from $A$-mod to the category of vector spaces $\mathcal{V}_{\text {ect }}$. We now state two lemmas. The proof of the first is straightforward and we leave it to the reader. 

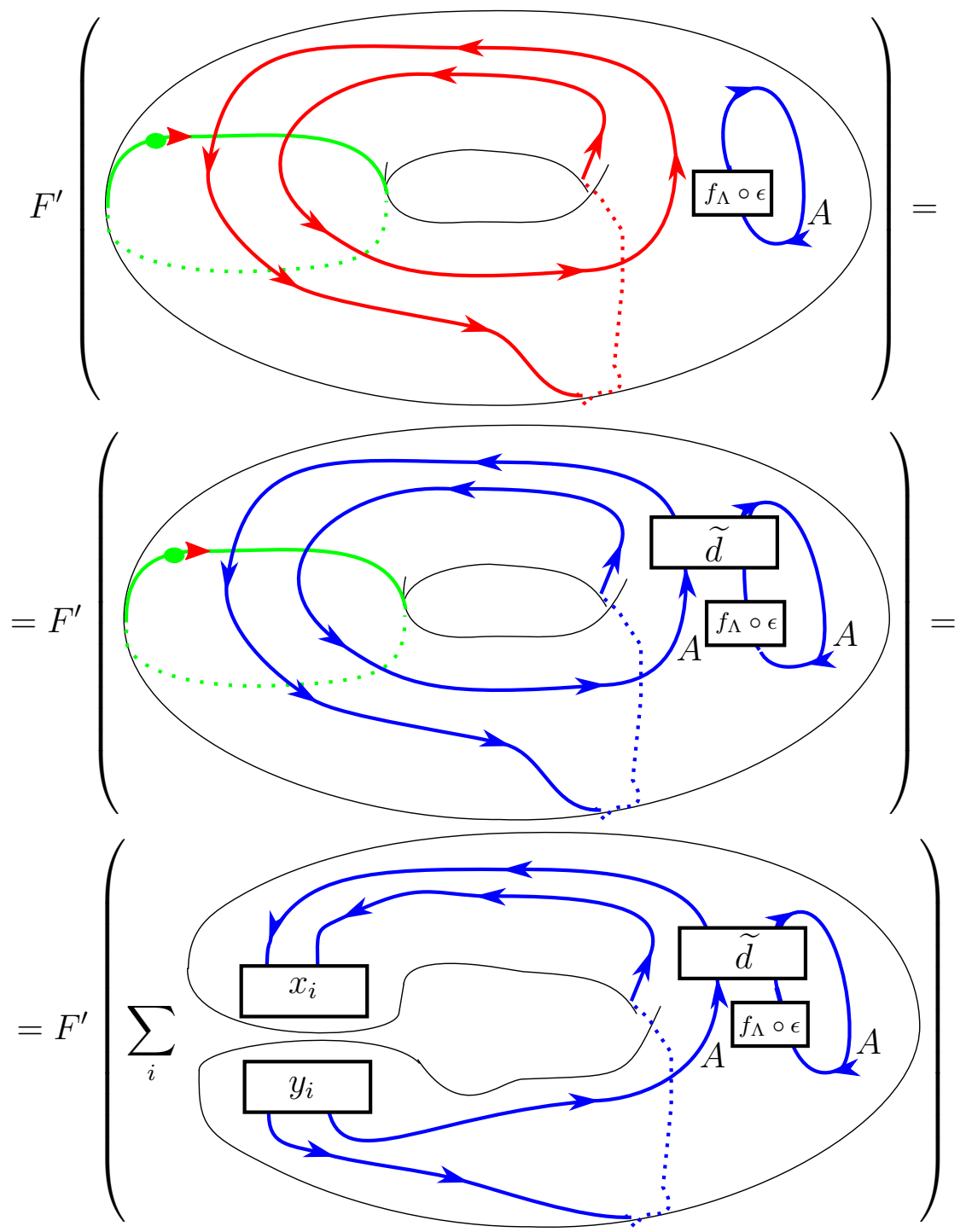

Figure 4 . Computation of $\mathcal{K}_{A \text {-mod }}$ for the lens space $L(2,1)$. The first equality is obtained using the chromatic morphism to transform the red circle into a blue graph. Then cutting along the meridian (depicted on the left) we obtain a graph $\Gamma^{\prime} \subset \partial B^{3}$. By definition, $\mathcal{K}_{A-\bmod }(L(2,1))=F^{\prime}\left(B^{3} ; \Gamma^{\prime}\right)$. 
Lemma 6.5. We have the following commutative diagram:

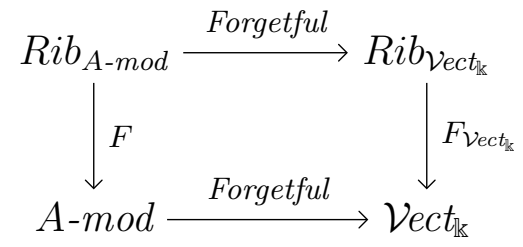

where the horizontal arrows represent the forgetful functors and the vertical arrows represent the pivotal Penrose functors from the categories of planar colored ribbon graphs to $A$-mod and Vect $t_{\mathbb{k}}$ respectively.

The next lemma says that evaluating red circles with the chromatic morphism is essentially the integral. This lemma directly follows from the definition of the chromatic morphism and Formula (13).

Lemma 6.6. For all $x \in A$ the following equality holds in $\mathcal{V}_{\text {ect }}$ :

$$
\left(\overleftarrow{\mathrm{ev}}_{A} \otimes \operatorname{Id}_{A}\right)\left(\operatorname{Id}_{A^{*}} \otimes L_{x} \otimes \operatorname{Id}_{A}\right)\left(\operatorname{Id}_{A^{*}} \otimes \widetilde{d}\right)\left(\overrightarrow{\operatorname{cov}}_{A} \otimes \operatorname{Id}_{A}\right)=\lambda(x) \operatorname{Id}_{A}
$$

This equality is represented pictorially as

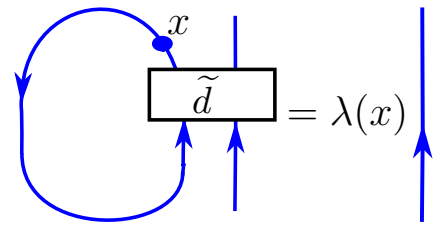

where all strands are colored with $A$ and the thick dot is labeled with $x$.

Now we can compute $\mathcal{K}_{A \text {-mod }}(M)$ (for involutive $A$ ) and prove Theorem 2.6. By Formula (14), we need to compute $F^{\prime}\left(B^{3}, \Gamma^{\prime}\right)$. Let $T_{A}$ be a $(1,1)$-ribbon graph whose closure is $\Gamma^{\prime}$. Formula (8) implies that

$$
F^{\prime}\left(B^{3}, \Gamma^{\prime}\right)=\mathrm{t}_{A}\left(F\left(T_{A}\right)\right) \text {. }
$$

Recall the $A$-mod morphism $f_{\Lambda} \circ \epsilon$ defined at the beginning of Section 6.2. Since $\mathrm{t}_{A}\left(f_{\Lambda} \circ \epsilon\right)=1$, Theorem 2.6 follows from the next claim:

Claim 6.7. $F\left(T_{A}\right)=\mathrm{Ku}_{A}(M) \cdot\left(f_{\Lambda} \circ \epsilon\right)$.

Proof of Claim 6.7. Lemma 6.5 implies that

$$
\operatorname{Forgetful}\left(F\left(T_{A}\right)\right)=F_{\text {Vect }_{\mathbb{k}}}\left(\operatorname{Forgetful}\left(T_{A}\right)\right) .
$$

Thus, it suffices to prove that

$$
F_{\text {Vect }_{\mathrm{k}}}\left(\operatorname{Forgetful}\left(T_{A}\right)\right)=\mathrm{Ku}_{A}(M) \cdot \operatorname{Forgetful}\left(f_{\Lambda} \circ \epsilon\right)
$$

since Forgetful is a faithful functor. In the rest of the proof, we work in the category $\mathcal{V}_{\mathrm{ect}}$. This allows us to consider the left multiplication by an 


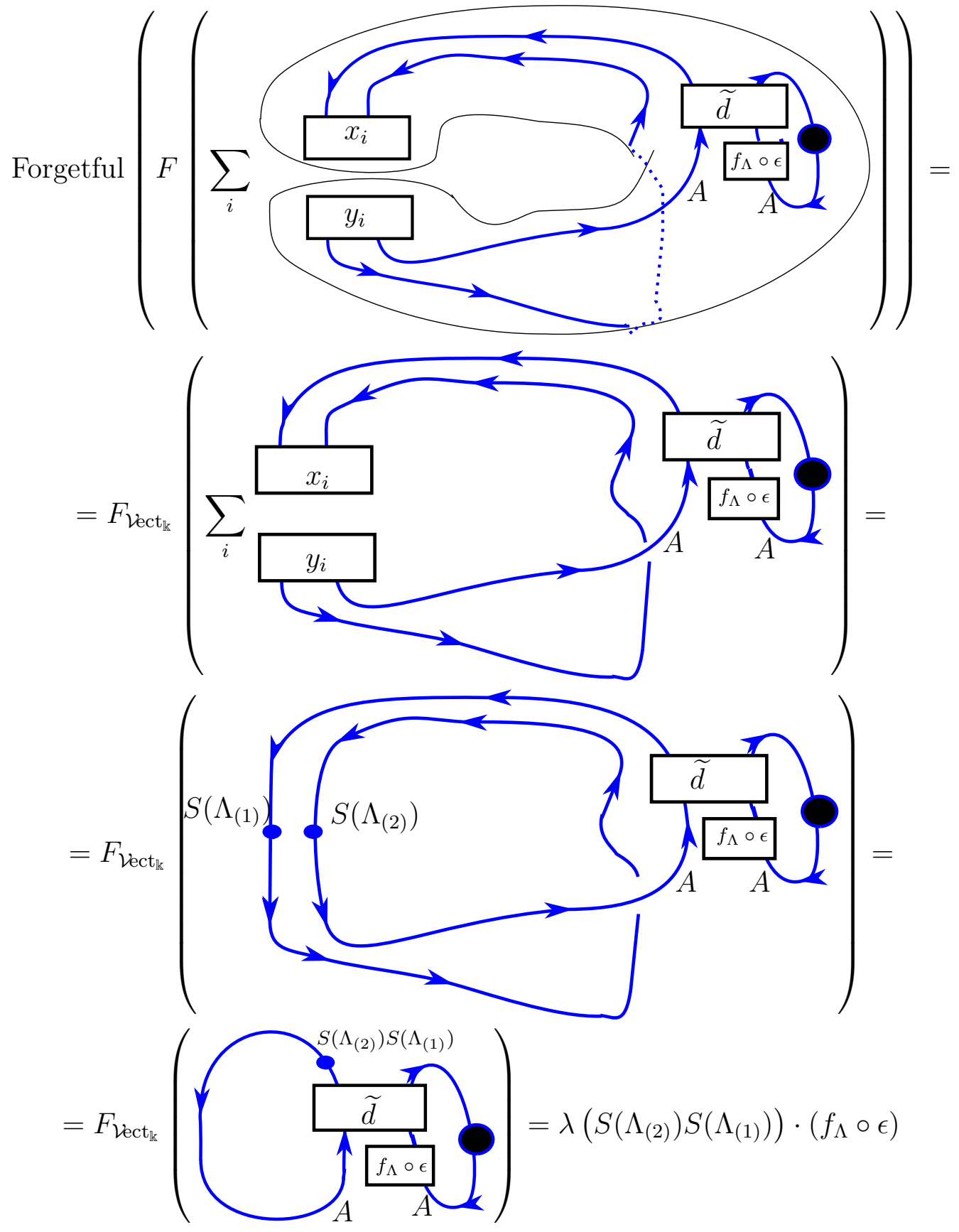

Figure 5. In Figure 4 we showed $\mathcal{K}_{A \text {-mod }}(L(2,1))=$ $F^{\prime}\left(B^{3}, \Gamma^{\prime}\right)$; here we continue this computations when $A$ is involutive. The first drawing depicts the 1-1 tangle obtained by cutting $\Gamma^{\prime}$ along the black disc. In the first equality, we pass to the category Vect $_{\mathrm{k}}$ using Lemma 6.5. Since Vect k $_{\mathrm{k}}$ has a symmetric braiding, the crossings make sense. The third equality re-expresses the cutting through beads (see Lemma 6.2). Finally, we collect the beads and apply Lemma 6.6. 
element of $A$ as a morphism in Vect $_{\mathbb{k}}$. To simplify notation we identify each morphism in $A$-mod with its underlying linear map.

Recall that cutting at one of the $\alpha$-circles, we replace the blue circles crossing it with an ordered pair of coupons filled with a sum $\sum_{i} x_{i} \otimes_{\mathbb{k}} y_{i}$, see Formula (2). By Lemma 6.2 the corresponding morphism is the left action by $\Lambda$. We provide each circle $\alpha_{i}$ with an orientation and a base point. As above, we use the chromatic morphism to change all red circles $\left\{\beta_{i}\right\}$ into blue graphs (this yields an orientation on each $\beta_{i}$ ). Instead of cutting the Heegaard surface along the circles $\left\{\alpha_{i}\right\}$ we decorate the blue graph with certain elements of $A$ called beads. Namely, each circle $\alpha_{j}$ intersects the upper circles $\left\{\beta_{i}\right\}$ transversely, and we let $c_{1}, \ldots, c_{m}$ be these intersections enumerated in the order they are met when traveling along $\alpha_{j}$ in the positive direction starting and ending in the base point. For $k=1, \ldots, m$, set $p_{k}=0$ if the tangent vectors of $\alpha_{j}$ and the circle $\beta_{i}$ meeting $\alpha_{j}$ at $c_{k}$ (taken in this order) form a positively oriented basis in the tangent space at $c_{k}$; otherwise set $p_{k}=1$. We assign to each $c_{k}$ the "bead" $S^{p_{k}}\left(\Lambda_{(k)}\right)$ where $\Delta^{m}(\Lambda)=$ $\Lambda_{(1)} \otimes \ldots \otimes \Lambda_{(m)}$.

Since Vect $_{\mathbb{k}}$ has a trivial ribbon structure, the left hand side of Formula (15) depends only on the abstract graph of $T_{A}$ or, equivalently, on $\Gamma^{\prime}$. Thus, we can compute $F_{\text {Vect }_{\mathrm{k}}}\left(\right.$ Forgetful $\left.\left(T_{A}\right)\right)$ as follows. Each upper circle $\beta_{i}$ has an orientation and a base point (determined by where the chromatic morphism is applied). Starting at this point and following the orientation we collect the beads to obtain a word $a_{i}$ of $A$ written from right to left. Doing this for all upper circles we obtain $g$ beads: $a_{1}, \ldots, a_{g}$. For each $\beta_{i}$, apply Lemma 6.6 where $x$ is the bead $a_{i}$ to obtain

$$
F_{\text {Vect }_{\mathrm{k}}}\left(\operatorname{Forgetful}\left(T_{A}\right)\right)=\lambda\left(a_{1}\right) \lambda\left(a_{2}\right) \ldots \lambda\left(a_{g}\right) \cdot\left(f_{\Lambda} \circ \epsilon\right) .
$$

For an example of this computation see Figure 5. But since $A$ is involutive, the definition of the Kuperberg invariant implies that

$$
\mathrm{Ku}_{A}(M)=\lambda\left(a_{1}\right) \lambda\left(a_{2}\right) \ldots \lambda\left(a_{g}\right) .
$$

This implies our claim above and completes the proof of the theorem.

\section{Proof of Theorem 2.7}

In this section we will use notation of Section 2.4. Let $\mathcal{T}$ be a triangulation of a closed connected oriented 3-manifold $M$ and let $t$ be a maximal tree of edges of $\mathcal{T}$. Clearly, $t$ contains all the vertices of $\mathcal{T}$. Let $H_{\beta}$ be a regular neighborhood of the 1 -skeleton of $\mathcal{T}$ in $M$ and let $H_{\alpha}=M \backslash\left(\operatorname{Int}\left(H_{\beta}\right)\right.$. Then $M=H_{\alpha} \cup H_{\beta}$ is a Heegaard splitting of $M$. The $\beta$-circles are meridians of the edges of $\mathcal{T}$ not in the tree $t$ and the $\alpha$-circles bound discs lying in the 2-dimensional faces of $\mathcal{T}$. As in Section 5.2 this Heegaard diagram gives 
a bichrome handlebody graph $\left(H_{\alpha}, \Gamma\right)$ where the red graph consists of the $\beta$-circles.

By the definition of the chromatic morphism, the value of a red unknot is the dimension $\mathcal{D} \neq 0$ of $\mathscr{C}$ (see Formula $(7)$ ). Thus, the value of $F^{\prime}\left(H_{\alpha}, \Gamma\right.$ ) does not change if we multiply it by $1 / \mathcal{D}$ and at the same time add a red unknot to the bichrome handlebody graph $\left(H_{\alpha}, \Gamma\right)$. With this in mind, we construct a new bichrome handlebody graph as follows. Starting with $\left(H_{\alpha}, \Gamma\right)$ place a red unknot on the boundary of the neighborhood of each edge of the tree $t$. Let $e$ be a leaf of $t$, i.e. an edge of $t$ such that one of its vertices is not adjacent to other edges of $t$. Then we can slide the new red unknot associated to $e$ over the red meridians of all other edges adjacent to this vertex. The resulting unknot is a red meridian around $e$. Continuing this process on the leaves of $t \backslash\{e\}$ etc., we obtain a bichrome handlebody graph $\left(H_{\alpha}, \Gamma^{\prime}\right)$ such that each edge of $\mathcal{T}$ has a red meridian going around this edge. Using this construction and the sliding property of $F^{\prime}$ for red circles, we get

$$
\mathcal{K}_{\mathscr{C}}(M)=F^{\prime}\left(H_{\alpha}, \Gamma\right)=\frac{1}{\mathcal{D}^{v-1}} F^{\prime}\left(H_{\alpha}, \Gamma^{\prime}\right)
$$

where $v$ is the number of vertices of $\mathcal{T}$.

Now we compute $F^{\prime}\left(H_{\alpha}, \Gamma^{\prime}\right)$. Use the chromatic morphism to make all the red circles blue. By definition of the chromatic morphism, each red circle is changed to a blue circle colored with $G$ or, equivalently, colored with the weighted $\operatorname{sum} \sum_{i}$ qdim $\left(S_{i}\right) S_{i}$ (note this happens with a meridian of each edge of $\mathcal{T}$ ). Furthermore, cutting along the discs formed by the 2-dimensional faces of $\mathcal{T}$ we obtain a set of spherical tetrahedra (indexed by the set of tetrahedra of $\mathcal{T}$ ) whose edges are all colored by $G$ and whose four 3-legged coupons are filled with morphisms coming from the cutting. Since $G$ splits as an orthogonal direct sum of simple objects, each of these spherical tetrahedra is a sum indexed by colorings of the edges of $T$ by elements of the set $\left\{S_{i}\right\}$. Moreover, each component of this sum is proportional to the corresponding $6 j$-symbol. Therefore,

$$
F^{\prime}\left(H_{\alpha}, \Gamma^{\prime}\right)=\mathcal{D}^{v} \operatorname{TV}_{\mathscr{C}}(M)
$$

where $\mathrm{TV}_{\mathscr{C}}(M)$ is the Turaev-Viro invariant of $M$ computed on the triangulation $\mathcal{T}$ of $M$, see [26]. Combining this with Formula (16), we obtain that $\mathcal{D}^{-1} \mathcal{K}_{\mathscr{C}}(M)=\operatorname{TV}_{\mathscr{C}}(M)$.

\section{REFERENCES}

1. J. Barrett, B. Westbury - Invariants of Piecewise-Linear 3-Manifolds. Transactions of the American Mathematical Society 348 (10), 3997-4022.

2. J. Barrett, B. Westbury - Spherical categories. Adv. Math. 143 (1999), 357-375.

3. A. Beliakova, C. Blanchet, A. Gainutdinov - Modified Trace is a Symmetrised Integral. arXiv:1801.00321. 
4. A. Beliakova, C. Blanchet, N. Geer - Logarithmic Hennings Invariants for Restricted Quantum $\mathfrak{s l}(2)$. arXiv:1705.03083.

5. D. Bulacu, S. Caenepeel - Integrals for (dual) quasi-Hopf algebras. Applications. J. Algebra 266 (2) (2003) 552-583.

6. D. Bulacu, S. Caenepeel - On integrals and cointegrals for quasi-Hopf algebras. J. Algebra 351 (2012), 390-425.

7. F. Costantino, N. Geer, B. Patureau-Mirand - Quantum Invariants of 3-Manifolds via Link Surgery Presentations and Non-Semi-Simple Categories. Journal of Topology 7 (2014), no. 4, 1005-1053.

8. M. De Renzi, N. Geer, B. Patureau-Mirand - Renormalized Hennings Invariants and 2+1-TQFTs. Comm. Math. Phys. 362 (2018), no. 3, 855-907.

9. P. Etingof, D. Nikshych, V. Ostrik - On fusion categories. Ann. of Math. (2) 162 (2005), no. 2, 581-642.

10. N. Geer, J. Kujawa, B. Patureau-Mirand - Generalized trace and modified dimension functions on ribbon categories. Selecta Mathematica, New Series 17 (2011), no. 2, 453-504.

11. N. Geer, J. Kujawa, B. Patureau-Mirand - Ambidextrous objects and trace functions for nonsemisimple categories. Proc. Amer. Math. Society 141 (2013), no. 9, 29632978.

12. N. Geer, J. Kujawa, B. Patureau-Mirand - M-traces in (non-unimodular) pivotal categories. preprint, arXiv:1809.00499.

13. N. Geer, B. Patureau-Mirand, V. Turaev - Modified 6j-Symbols and 3-Manifold invariants. Advances in Mathematics 228 (2011), no. 2, 1163-1202.

14. N. Geer, B. Patureau-Mirand, A. Virelizier, - Traces on ideals in pivotal categories. Quantum Topology 4 (2013), no. 1, 91-124.

15. R. Kashaev, A. Virelizier - Generalized Kuperberg invariants of 3-manifolds. arxiv:1805.00413.

16. G. Kuperberg - Involutory Hopf algebras and 3-manifold invariants. Internat. J. Math. 2 (1991), no. 1, 41-66.

17. G. Kuperberg - Non-involutory Hopf algebras and 3-manifold invariants. Duke Math J. 84 (1996) (1) 83-129.

18. F. Hausser, F. Nill - Integral theory for quasi-Hopf algebras. preprint, math.QA/9904164.

19. H. Masur, Y. Minsky - Quasiconvexity in the curve complex. in "In the tradition of Ahlfors and Bers, III", ed. W. Abikoff, A. Haas, Contemp. Math. 355, Amer. Math. Soc. (2004) 309-320.

20. F. Panaite, F. Van Oystaeyen - Existence of integrals for finite dimensional quasi-Hopf algebras. Bull. Belg. Math. Soc. Simon Stevin 7 (2000), no. 2, 261-264.

21. D. Radford - Hopf Algebras. Series on Knots and Everything: Volume 49 (2011).

22. M Scheunert, R. B. Zhang - Invariant integration on classical and quantum Lie supergroups. J. Math. Phys. 42 (2001), no. 8, 3871-3897.

23. M Scheunert, R. B. Zhang - Integration on Lie supergroups: a Hopf algebra approach. J. Algebra 292 (2005), 324-342.

24. K. Shimizu - Integrals for finite tensor categories. arXiv:1702.02425.

25. J. Singer - Three dimensional manifolds and their Heegaard diagrams. Trans. Amer. Math. Soc. 35 (1933), 88-111.

26. V. Turaev - Quantum invariants of knots and 3-manifolds. de Gruyter Studies in Mathematics, 18. Walter de Gruyter \& Co., Berlin, (1994). 
27. V. Turaev, A. Virelizier, - Monoidal categories and topological field theory. Progress in Mathematics, 322. Birkhäuser/Springer (2017).

28. V. Turaev, O. Viro - State sum invariants of 3-manifolds and quantum $6 j$-symbols. Topology 31 (1992), no. 4, 865-902.

Institut de Mathématiques de Toulouse, 118 route de Narbonne, Toulouse F-31062

E-mail address: francesco.costantino@math.univ-toulouse.fr

Mathematics \& Statistics, Utah State University, Logan, Utah 84322, USA

E-mail address: nathan.geer@gmail.com

UMR 6205, LMBA, université de Bretagne-Sud, université européenne de Bretagne, BP 573, 56017 Vannes, France

E-mail address: bertrand.patureau@univ-ubs.fr

Department of Mathematics, Indiana University, Rawles Hall, 831 East 3RD ST, BLOOMINGTON, IN 47405, USA

E-mail address: vtouraev@indiana.edu 